\title{
Kondo effect in a carbon nanotube with spin-orbit interaction and valley mixing: A DM-NRG study
}

\author{
Davide Mantelli a,*, Cătălin Paşcu Moca ${ }^{\mathrm{b}, \mathrm{c}}$, Gergely Zaránd ${ }^{\mathrm{b}}$, Milena Grifoni ${ }^{\mathrm{a}}$ \\ a Institut für Theoretische Physik, Universität Regensburg, 93040 Regensburg, Germany \\ ${ }^{\mathrm{b}}$ Department of Theoretical Physics, Institute of Physics, Budapest University of Technology and Economics, HU-1521 Budapest, Hungary \\ c Department of Physics, University of Oradea, 410087 Oradea, Romania
}

\section{A R T I C L E I N F O}

\section{Article history:}

Received 18 July 2015

Received in revised form

19 November 2015

Accepted 19 November 2015

Available online 2 December 2015

PACS:

73.63.Fg

73.21.La

72.15.Qm

Keywords:

Kondo effect

Carbon nanotubes

Strong coupling

Density matrix numerical renormalization

group

\begin{abstract}
A B S T R A C T
We investigate the effects of spin-orbit interaction (SOI) and valley mixing on the transport and dynamical properties of a carbon nanotube (CNT) quantum dot in the Kondo regime. As these perturbations break the pseudo-spin symmetry in the CNT spectrum but preserve time-reversal symmetry, they induce a finite splitting $\Delta$ between formerly degenerate Kramers pairs. Correspondingly, a crossover from the SU (4) to the SU(2)-Kondo effect occurs as the strength of these symmetry breaking parameters is varied. Clear signatures of the crossover are discussed both at the level of the spectral function as well as of the conductance. In particular, we demonstrate numerically and support with scaling arguments that the Kondo temperature scales inversely with the splitting $\Delta$ in the crossover regime. In presence of a finite magnetic field, time reversal symmetry is also broken. We investigate the effects of both parallel and perpendicular fields (with respect to the tube's axis) and discuss the conditions under which Kondo revivals may be achieved.
\end{abstract}

(c) 2015 Elsevier B.V. All rights reserved.

\section{Introduction}

The Kondo effect [1] is a hallmark of strongly correlated electron physics. Its observation in quantum dot set-ups is ubiquitous and reveals precious information on the underlying symmetries of the quantum dot system and on the corresponding degeneracies of its spectrum. Specifically, electrons in carbon nanotubes (CNTs) possess a spin and a pseudo-spin degree of freedom [2], the latter originating from the presence of two inequivalent Dirac points in the underlying graphene hexagonal lattice. In the absence of spinorbit interaction, and considering only transverse quantization, the CNT's Hamiltonian is invariant under time-reversal and pseudo-

\footnotetext{
* Corresponding author.

E-mail address: davide.mantelli@physik.uni-regensburg.de (D. Mantelli).
}

spin reversal symmetries, and thus a quadruplet of degenerate levels is associated with a given longitudinal momentum. In this case, the four-fold degeneracy may lead to the occurrence of the so-called SU(4) - Kondo effect at low temperatures [3-7]. In order to see this exotic Kondo resonance it is important, however, that both spin and pseudo-spin quantum numbers be conserved during tunneling (or reflection), as a mixing of these degrees of freedom can result in a more conventional SU(2) Kondo effect [8]. For CNT devices where parts of the tube act as leads (see Fig. 1), such a situation can be realized, and the peculiar features associated to the presence of both spin and orbital degrees of freedom can be probed in finite magnetic fields [6,9]. Recently, SU(4) Kondo physics, also originating from coupled spin and orbital degrees of freedom, could be engineered in double-quantum dot based devices [10]. 


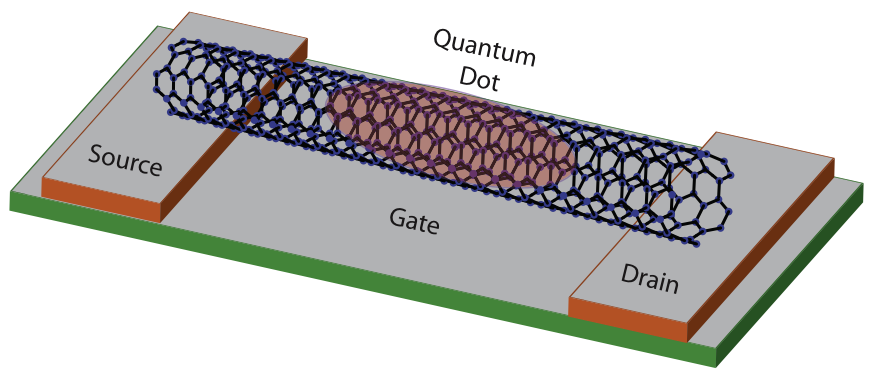

Fig. 1. Sketch of the setup. The carbon nanotube is coupled to two external contacts, source and drain. The quantum dot formed in the nanotube is indicated by the shaded area. The electrostatic state of the dot is capacitively controlled by a back-gate.

In a more realistic description of a CNT though, pseudo-spin symmetry breaking contributions, like the curvature induced spin-orbit interaction (SOI) $[11,12]$ or valley mixing due to scattering off the boundaries [13,14] or to disorder [12], should be included. As a consequence, the fourfold degeneracy is broken and, for a given value of the longitudinal momentum, the spectrum of an isolated CNT quantum dot consists of two pairs of degenerate Kramers pairs, with splitting provided by the combined effects of SOI and valley mixing [15]. In this situation, upon increasing the SOI strength or the valley mixing, a crossover from the SU(4)- Kondo state involving both Kramers pairs, to the more standard SU(2) Kondo regime is expected [16-18].

Despite the considerable amount of experiments reporting Kondo behavior in CNTs, [6,9,18-24], the combined effect of SOI, valley mixing and the impact of applied magnetic fields on the SU(4) to SU(2) crossover have only been addressed within a field theoretical effective Keldysh action approach [18]. A numerically exact investigation of the highly intricate crossover is thus very desirable.

In this work we study the dynamical and linear transport properties of CNT-based Kondo quantum dots by means of the Density Matrix-Numerical Renormalization Group (DM-NRG) method [16,25-27]. We focus on the SU(4) to SU(2) crossover induced by finite SOI and valley mixing, and study the influence of magnetic fields parallel or perpendicular to the CNT's axis. At zero magnetic field, it is not possible to distinguish at the level of the spectral function or of the linear conductance among the two symmetry breaking effects. Here, what matters is the amplitude $\Delta$ of the total inter-Kramers splitting. We determine here the energy scales for the cross-over region and demonstrate that the Kondo temperature scales inversely with $\Delta$, in agreement with previous analytical predictions $[28,29]$.

At finite fields, the behavior of the Kondo resonance is strongly influenced by the relative strength of the SOI and valley mixing contributions, as well as by the direction of the applied field. In fact, a major effect of the curvature induced SOI is to set as spin quantization axis the tube's axis and to lock spin and valley

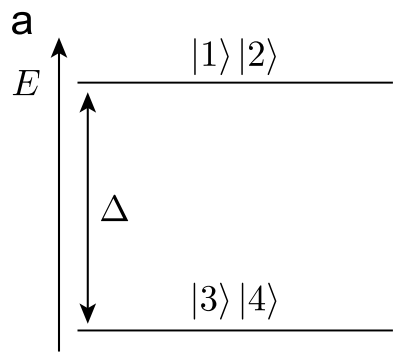

b

Fig. 2. (a) Energy level scheme involving two Kramers doublets separated by the energy $\Delta$. (b) Connection among the energy levels established by the symmetry operations $\hat{\mathcal{T}}, \hat{\mathcal{P}}$ and $\hat{C}$. degrees of freedom [11]. Valley mixing instead does not act on the spin degree of freedom, but induces a rotation in valley space $[13,15]$. Thus, in parallel field the spin is still a good quantum number. Magnetic field induced Aharnov-Bohm contributions dominate over Zeeman effects at small fields, due to the large orbital moment of the nanotubes [2], which enables one to clearly resolve the splitting [23] and rejoining [15,18] of a Kramers pair also at low fields. In perpendicular fields, in contrast, the spin is no longer a good quantum number; rather the simultaneous presence of SOI and valley mixing implies a full entanglement of orbital and spin degrees of freedom. In this case it is convenient to classify virtual Kondo transitions in terms of the discrete operations related to the time reversal and pseudo-spin reversal operators [18]. These considerations are nicely confirmed by our simulations and reflected, in particular, at the level of the linear conductance in the occurrence of Kondo revivals: at specific values of the magnetic field, which depend on the field direction, valley mixing strength and on the number of charges trapped in the dot, a Kondo resonance can be restored near avoided level crossings.

The paper is organized as follows. We present our model Hamiltonian for CNTs in Section 2.1, and give a brief analysis of the symmetries of the system in Section 2.2. Dynamical and transport properties are discussed in Sections 3 and 4. We extend our discussion in Section 5 by including the effects of an applied magnetic field parallel (Section 5.1) or perpendicular (Section 5.2) to the CNT's axis. Our conclusions are summarized in Section 6.

\section{Theoretical framework}

\subsection{Model Hamiltonian}

The setup we consider consists of a CNT quantum dot coupled to two external leads (see the sketch in Fig. 1). We focus on a single longitudinal mode (also known as "shell"), and correspondingly, describe the CNT by an extended Anderson impurity model $[1,15]$, consisting of a pair of interacting Kramers doublets. We denote by $\varepsilon_{j}$ the energies of the four levels $(j=\{1,2,3,4\})$, and by $\hat{n}_{j}=\hat{d}_{j}^{\dagger} \hat{d}_{j}$ their occupation. In what follows, we shall refer to this basis as the Kramers basis (see Fig. 2(a)). Each of the four levels can accommodate one electron and, with a good approximation, these electrons interact with each other through a strong and level-independent on-site interaction $U$. In this basis, the CNT Hamiltonian takes the form

$\hat{H}_{\mathrm{CNT}}=\sum_{j=1}^{4} \varepsilon_{j} \hat{n}_{j}+U \sum_{j<j^{\prime}}^{4} \hat{n}_{j} \hat{n}_{j^{\prime}}$.

In the absence of the spin-orbit interaction and valley mixing, $\Delta_{\mathrm{SO}}=0$ and $\Delta_{\mathrm{KK}^{\prime}}=0$, the CNT's Hamiltonian is invariant under time-reversal and valley-reversal [2]. These operations are represented by the two antiunitary operators $\hat{\mathcal{T}}$ and $\hat{\mathcal{P}}$, respectively [18], and yield a fourfold degenerate spectrum of the CNT, $\varepsilon_{j} \equiv \varepsilon_{\mathrm{d}}$. Correspondingly,the CNT Hamiltonian is SU(4) invariant. In what follows, we shall label states such that $(1,2)$ and $(3,4)$ form Kramers pairs, while $(1,4)$ and $(2,3)$ are pairs associated with the $\hat{\mathcal{P}}$ symmetry. Notice that a third unitary operator $\hat{C}=\hat{\mathcal{P}} \hat{\mathcal{T}}^{-1}$ linking the remaining pairs $(1,3)$ and $(2,4)$ can also be constructed from $\hat{\mathcal{T}}$ and $\hat{\mathcal{P}}$ (see Fig. 2(b)). A finite $\Delta=\sqrt{\Delta_{\mathrm{SO}}^{2}+\Delta_{\mathrm{KK}}^{2}}$ breaks the $\hat{\mathcal{P}}$ symmetry and, correspondingly, also the SU(4) symmetry (see Appendix A for details on how these states and the symmetry operations are constructed). Since time-reversal symmetry is preserved, the on-site energies remain twofold degenerate, $\varepsilon_{1}=\varepsilon_{2}=\varepsilon_{\mathrm{d}}+\Delta / 2$ and $\varepsilon_{3}=\varepsilon_{4}=\varepsilon_{\mathrm{d}}-\Delta / 2$ (see Fig. 2(a)). Notice that 
a finite $\Delta$ plays the same role as a magnetic field on the $\mathcal{P}-$ and $C$-pairs, such that conjugation relations among energy levels exist: $\varepsilon_{1}(\Delta)=\varepsilon_{4}(-\Delta)$, and similarly for the other couples.

In a CNT, with a good approximation, each nanotube level couples to independent channels in the leads, and their tunnel coupling can thus be described by the Hamiltonian

$\hat{H}_{\text {tun }}=\sum_{j} V_{j} \sqrt{\rho_{0}} \int \mathrm{d} \varepsilon \hat{a}_{\varepsilon j}^{\dagger} \hat{d}_{j}+$ h. c..

Here, instead of the original left/right operators $\hat{c}_{\varepsilon j, \mathrm{~L} / \mathrm{R}}^{\dagger}$ for the leads, we introduced the symmetric and antisymmetric combinations

$\left(\begin{array}{c}\hat{a}_{\varepsilon j} \\ \hat{b}_{\varepsilon j}\end{array}\right)=\left(\begin{array}{cc}\cos \gamma_{j} & \sin \gamma_{j} \\ -\sin \gamma_{j} & \cos \gamma_{j}\end{array}\right)\left(\begin{array}{c}\hat{c}_{\varepsilon j, \mathrm{~L}} \\ \hat{c}_{\varepsilon j, \mathrm{R}}\end{array}\right)$,

and the corresponding effective tunneling amplitude $V_{j} \equiv \sqrt{V_{j \mathrm{~L}}^{2}+V_{j \mathrm{R}}^{2}}$ and asymmetry parameter $\gamma_{j} \equiv \arctan V_{j \mathrm{R}} / V_{j \mathrm{~L}}$. The leads are assumed to be non-interacting with a constant density of states per flavor $\rho(\omega)=\rho_{0}=1 / 2 W$, and a bandwidth $2 W$. They are described by the Hamiltonian

$\hat{H}_{\text {lead }}=\sum_{j} \int_{-W}^{W} d \varepsilon \varepsilon\left(\hat{a}_{\varepsilon j}^{\dagger} \hat{a}_{\varepsilon j}+\hat{b}_{\varepsilon j}^{\dagger} \hat{b}_{\varepsilon j}\right)$.

Notice that only the $\hat{a}_{\varepsilon j}$ channel couples to the dot, while channel $\hat{b}_{\varepsilon j}$ remains completely decoupled in equilibrium. The total Hamiltonian

$\hat{H}=\hat{H}_{\text {CNT }}+\hat{H}_{\text {tun }}+\hat{H}_{\text {lead }}$

captures the essential physics of our set-up and, under equilibrium conditions, can be solved using Wilson's NRG method [25].

\subsection{Global symmetries}

Let us now discuss the continuous symmetries of the Hamiltonian (4). These symmetries are extremely useful, since they allow for an efficient numerical treatment of the problem. Throughout this paper, we shall focus on the simplest but physically relevant case of

$V_{j} \equiv V, \quad \gamma_{j} \equiv \gamma$.

In this case, for $\Delta=0$, the total $\mathrm{SU}(4)$-spin operator

$\hat{\mathbf{J}}^{\mathrm{SU}(4)}=\frac{1}{2} \sum_{j, j^{\prime}=1}^{4}\left(\hat{d}_{j}^{\dagger} \lambda_{j j^{\prime}} \hat{d}_{j^{\prime}}+\int \mathrm{d} \varepsilon \hat{a}_{\varepsilon j}^{\dagger} \lambda_{j j^{\prime}} \hat{a}_{\varepsilon j^{\prime}}\right)$

commutes with the Hamiltonian (4), and the SU(4) symmetrical Anderson model [7] is recovered. The $\lambda$ 's above denote the 15 generalized Gell Mann matrices or some other set of matrices defining the $\mathrm{SU}(4)$ representation, and the operators (5) satisfy the $\mathrm{SU}(4)$ Lie algebra.

Finite inter-valley scattering or spin-orbit field imply $\Delta \neq 0$, and break the $\mathrm{SU}(4)$ symmetry down to $\mathrm{SU}(2) \otimes \mathrm{SU}(2)$. The latter are generated by the usual $\mathrm{SU}(2)$ spin operators

$\hat{\mathbf{J}}_{\kappa}=\frac{1}{2} \sum_{j, j^{\prime} \in \kappa}\left(\hat{d}_{j}^{\dagger} \boldsymbol{\sigma}_{j j^{\prime}} \hat{d}_{j^{\prime}}+\int \mathrm{d} \varepsilon \hat{a}_{\varepsilon j}^{\dagger} \boldsymbol{\sigma}_{j j^{\prime}} \hat{a}_{\varepsilon j^{\prime}}\right)$,

acting on the two Kramers doublets $\kappa=(1,2)$ and $\kappa=(3,4)$ (see Fig. 2). Here, $\sigma=\left(\sigma_{x}, \sigma_{y}, \sigma_{z}\right)$ is the regular vector of the Pauli matrices. In addition to these $\mathrm{SU}(2)$ symmetries, the total charge is also conserved in each Kramers "channel"

\footnotetext{
${ }^{1}$ Quasiparticle operators are normalized to satisfy $\left\{\hat{a}_{\varepsilon j}, \hat{a}_{\varepsilon^{\prime} j^{\prime}}\right\}=\delta\left(\varepsilon-\varepsilon^{\prime}\right) \delta_{i j j^{\prime}}$.
}

$\hat{Q}_{\kappa}=\frac{1}{2} \sum_{j \in \kappa}\left(\hat{d}_{j}^{\dagger} \hat{d}_{j}-1 / 2+\int \mathrm{d} \varepsilon: \hat{a}_{\varepsilon j}^{\dagger} \hat{a}_{z j}:\right)$,

with :...: referring to normal ordering.

\section{Transport properties}

In this section we present the results for the spectral functions $A_{j}(\omega)$ of the operators $d_{j}^{\dagger}$ and evaluate the conductance across the dot under equilibrium conditions. The linear conductance can be computed directly within the NRG and is related to the equilibrium spectral function of the operators $d_{j}^{\dagger}$. It reads

$G(T)=\frac{e^{2}}{h} \sum_{j} \alpha_{j} \Gamma_{j} \pi \int_{-\infty}^{+\infty}\left(-\frac{\partial f(\omega, T)}{\partial \omega}\right) A_{j}(\omega)$,

with $\Gamma_{j}=\pi V_{j}^{2} \rho_{0}$ the usual broadening parameter, and $\alpha_{j}=4 \tan \gamma_{j} /\left(1+\tan \gamma_{j}\right)^{2}$ the asymmetry prefactor, which depends on the source and drain couplings and is in general smaller than one. In Eq. (8), $f(\omega, T)=(1+\exp (\omega / T))^{-1}$ (unit $k_{\mathrm{B}}=1$ ) is the Fermi-Dirac distribution function and $A_{j}(\omega)$ denotes the equilibrium spectral function of the $j$-th dot level

$A_{j}(\omega)=-\frac{1}{\pi} \operatorname{Im}\left\{G_{j, j}^{\mathrm{R}}(\omega)\right\}$,

with $G_{j, j^{\prime}}^{\mathrm{R}}(\omega)$ being the Fourier transform of the retarded Green's function $G_{j, j^{\prime}}^{\mathrm{R}}(t)=-i \Theta(t)\left\langle\left\{\hat{d}_{j}(t), \hat{d}_{j^{\prime}}^{\dagger}(0)\right\}\right\rangle$. To compute $A_{j}(\omega)$, we used the open-access Budapest DM-NRG code [30], that explicitly uses the symmetries of the system. As discussed above, for $\Delta=0$ the system exhibits $\mathrm{SU}(4)$ symmetry. Therefore, by tuning the parameter $\Delta$ from 0 to some large value $\Delta \gg W$, for a singly occupied longitudinal level, we can follow the crossover from the $\mathrm{SU}(4)-$ Kondo fixed point to the $\mathrm{SU}(2) \otimes \mathrm{SU}(2)$ one. In the next two subsections we shall study the manifestation of the crossover at the level of the spectral functions and of the linear conductance, respectively.

\subsection{Spectral functions}

The impact of the splitting $\Delta$ on the Kondo resonance is demonstrated in Figs. 3 and 4, where we display the total spectral function, $A_{\text {tot }}(\omega)=\sum_{j} A_{j}(\omega)$, for two specific values of $\varepsilon_{\mathrm{d}}$ and a relatively large ratio $U / \Gamma=50$. For $\Delta=0$ the occupation of the CNT levels is controlled by the "dimensionless gate voltage", $N_{\mathrm{g}}=\left(-\varepsilon_{\mathrm{d}}+U / 2+\Delta\right) /(U+\Delta / 2)$, taking on integer values $N_{\mathrm{g}}=k=1,2,3$ just in the middle of the Coulomb blockade valleys with $k$ particles on the CNT.

Fig. 3 displays the crossover from the SU(4) to the SU(2) regime for the case $N_{g}=1$ and several values of $\Delta$. By particle-hole symmetry, the spectral functions for $N_{g}=3$ are the mirror images of the $N_{g}=1$ spectral functions, and we do not discuss them in detail. In the limit $\Delta=0$, an SU(4) Kondo resonance arises due to quantum fluctuations of the ground state quadruplet. As expected [1], this resonance is pinned asymmetrically to the Fermi level, $\omega=0$. As soon as the splitting $\Delta$ becomes comparable to $T_{\mathrm{K}}^{\mathrm{SU}(4)}$ (extracted form the half width at half maximum of $A_{\text {tot }}(\omega)$ ), the spectral function maximum lowers and tends to be symmetrical around the Fermi energy. At the same time, two satellite peaks emerge at approximately $\pm \Delta$. These satellite peaks correspond to "electron-hole" excitations between the two Kramers pairs depicted in Fig. 2. Notice that the value of the spectral function does not change at the Fermi energy as the SU(4) resonance gradually turns into a symmetrical SU(2) Kondo resonance. This implies, according to Eq. (8), that in the $N_{g}=1$ valley the conductance at 


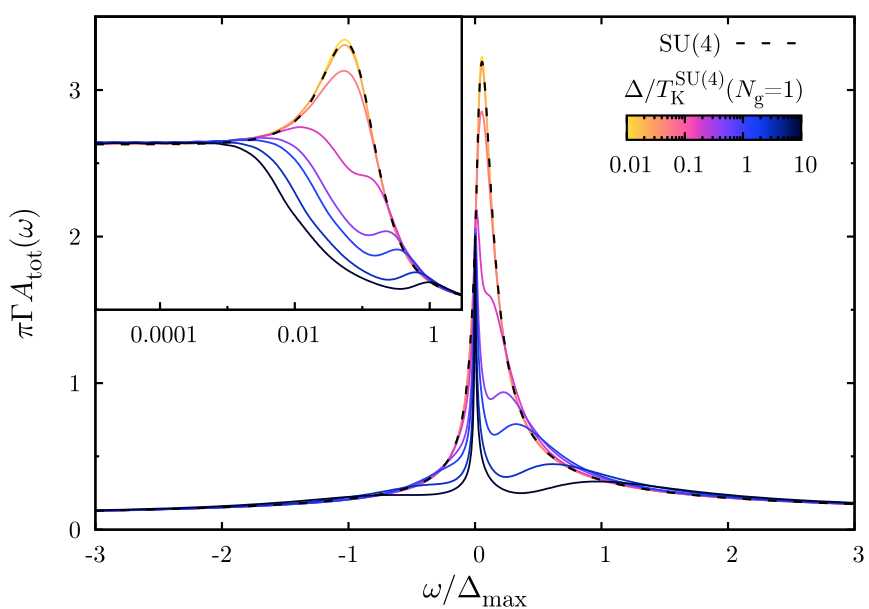

Fig. 3. The total spectral function $A_{\text {tot }}(\omega)$ as a function of frequency for different values of $\Delta$ (color scale in units of $T_{\mathrm{K}}^{\mathrm{SU}(4)}=T_{\mathrm{K}}(\Delta=0) \simeq 0.000429 \Gamma$ ). Other parameters were fixed to $U=W, \varepsilon_{\mathrm{d}}=-U / 2\left(N_{\mathrm{g}}=1\right), \Gamma=U / 50$ and $T=0$. Inset: The evolution of the Kondo peak for different values of $\Delta$. (For interpretation of the references to color in this figure caption, the reader is referred to the web version of this paper.)

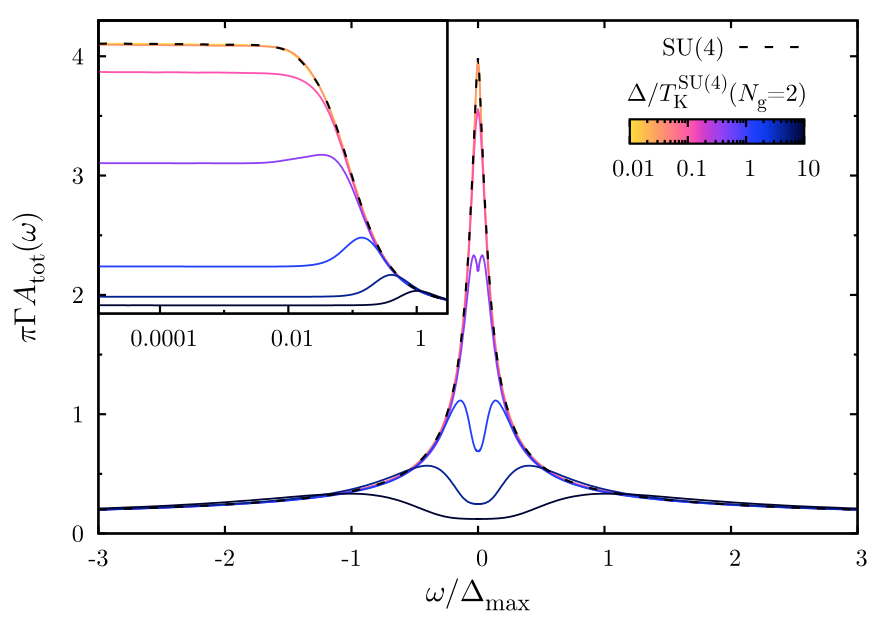

Fig. 4. The $T=0$ temperature total spectral function $A_{\text {tot }}(\omega)$ as a function of frequency for different values of $\Delta$ and at $\varepsilon_{\mathrm{d}}=-3 U / 2\left(N_{\mathrm{g}}=2\right)$. We have used $U=W$ and $\Gamma=U / 50$. (Color scale in units of $T_{\mathrm{K}}^{\mathrm{SU}(4)}=T_{\mathrm{K}}(\Delta=0) \simeq 0.000311 \Gamma$.) Inset: Evolution of the Kondo peak on a logarithmic scale for different values of $\Delta$. (For interpretation of the references to color in this figure caption, the reader is referred to the web version of this paper.)

$T=0$ is not suppressed when the $\mathrm{SU}(4)$ symmetry is broken $[3,4,7,8,17]$. However, as shown in the inset, the Kondo temperature is strongly reduced for $\Delta \gg T_{\mathrm{K}}^{\mathrm{SU}(4)}$.

The situation is dramatically different for $N_{\mathrm{g}}=2\left(\varepsilon_{\mathrm{d}}=-3 U / 2\right)$. At this value of $\varepsilon_{\mathrm{d}}$ there are two electrons on the CNT longitudinal shell. The Hamiltonian exhibits electron-hole symmetry, and the total spectral function is symmetrical for any value of $\Delta$. As a consequence of Friedel sum rule [31], the value of $A_{\text {tot }}(\omega=0)$ is twice as large as it was for $N_{\mathrm{g}}=1$. For $N_{\mathrm{g}}=2$, however, a splitting $\Delta \gg T_{\mathrm{K}}^{\mathrm{SU}(4)}$ eliminates the ground state degeneracy of the isolated $\mathrm{CNT}$, completely suppresses the Kondo resonance, and leads to the emergence of a "pseudogap" of width $\sim \Delta$ in $A_{\text {tot }}(\omega)$.

\subsection{Linear conductance}

The crossover features in the spectral function are also reflected in transport characteristics. In Fig. 5, we present the $T=0$ temperature linear conductance as a function of $N_{\mathrm{g}}$ for several values of the splitting $\Delta$. Similar to the spectral function, as a

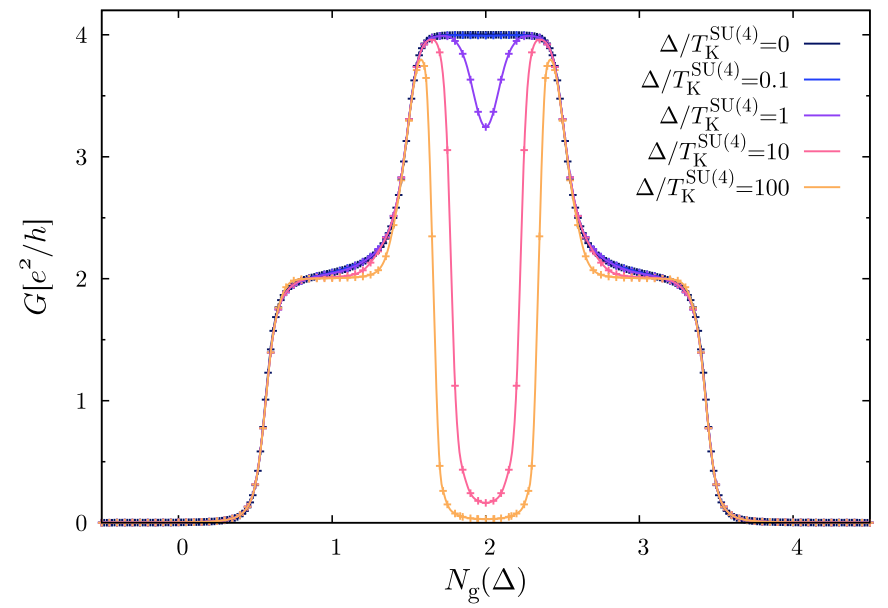

Fig. 5. Linear conductance as a function of $N_{\mathrm{g}}$ for different values of the splitting $\Delta$ $\left(T_{\mathrm{K}}^{\mathrm{SU}(4)}=T_{\mathrm{K}}\left(\Delta=0, N_{\mathrm{g}}=1\right)=0.000429 \Gamma\right)$. The parameters are $U=W, \Gamma=U / 50$ and $T=0$.

manifestation of the two electron SU(4) - Kondo state, the linear conductance also acquires its maximal value at the particle-hole symmetric point $N_{g}=2$ in the $\mathrm{SU}(4)$ symmetrical case, $\Delta=0$. This large conductance is, however, sensitive to $\Delta$ and is quickly suppressed for $\Delta \gg T_{\mathrm{K}}^{\mathrm{SU}(4)}$, as a consequence of the pseudogap appearing in the spectral function.

In contrast, for $N_{\mathrm{g}}=1$ and $N_{\mathrm{g}}=3$ a large $\Delta$ does not destroy the conductance at $T=0$ temperature, which remains close to $2 e^{2} / h$. Similar results for the conductance were previously obtained in Ref. [17] (see dashed curves in Fig. 4 therein). Here, tuning the ratio $U / \Gamma$ to larger values enables us to reach the $\mathrm{SU}(2)$ unitary limit in the $N_{\mathrm{g}}=1$ and $N_{\mathrm{g}}=3$ valleys, and an almost vanishing conductance at the particle-hole symmetric point in $N_{g}=2$ even at very large $\Delta / T_{\mathrm{K}}^{\mathrm{SU}(4)}$.

The origin of the $2 e^{2} / h$ conductance in the $N_{\mathrm{g}}=1,3$ cases is different in the limits $\Delta=0$ and $\Delta \gg T_{\mathrm{K}}^{\mathrm{SU}(4)}$. In the SU(4) limit, $\Delta \rightarrow 0$, incident conduction electrons pass through the quantum dot with probability $1 / 2$ in all four channels. In contrast, for $\Delta \rightarrow \infty$ the two empty levels and the corresponding channels do not conduct at all, while the other two have a perfect, unitary conductance due to the residual $\mathrm{SU}(2)$ Kondo effect. Since in these channels electrons pass through the dot with probability one, in this $\Delta \rightarrow \infty$ limit the linear conductance becomes noiseless [1].

In the light of Ref. [17], another useful way to visualize the crossover between the SU(4) and SU(2) regimes is to consider the temperature dependence of the linear conductance, shown for $N_{g}=1$ in Fig. 6. We extend here the analysis of Ref. [17] by exploring a larger range of ratios $\Delta / T_{\mathrm{K}}^{\mathrm{SU}(4)}$ (and $U / \Gamma=50$ ) which enables us to track the whole $\mathrm{SU}(4)$ to $\mathrm{SU}(2)$ crossover in more details. To explore universal scaling, we have rescaled the temperature by the Kondo temperature $T_{\mathrm{K}}^{\mathrm{HCM}}(\Delta)$, defined as the temperature at which the conductance is reduced to half of its $T=0$ temperature value [1]. For $\Delta$ much smaller than $T_{\mathrm{K}}^{\mathrm{HCM}}(\Delta)$, conductance curves show SU(4) universality and lie on the top of each other. As soon as $\Delta$ becomes comparable with $T_{\mathrm{K}}^{\mathrm{HCM}}(\Delta)$, however, universality is lost, the Kondo temperature lowers and a peak emerges at approximately $\Delta$. For very large values of $\Delta / T_{\mathrm{K}}^{\mathrm{HCM}}(\Delta)$, the curves become universal again, but they now follow a simple SU(2)-scaling. The two universal curves for $\Delta=0$ and $\Delta>U$ are associated with the SU(4) and SU(2) fixed points, and can be derived from the corresponding Kondo models (see Appendix B for further details). 


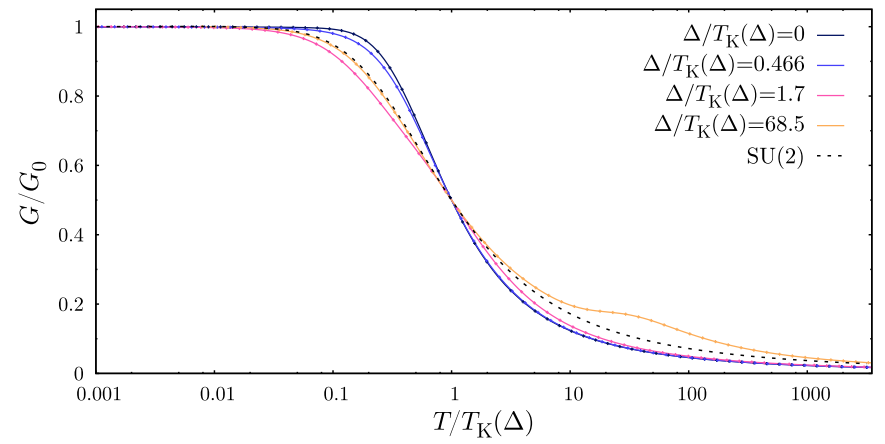

Fig. 6. DM-NRG result for the linear conductance as a function of $T / T_{\mathrm{K}}$ for different values of the splitting $\Delta$. The parameters are $U=W, \Gamma=U / 50, T=0$ and $\varepsilon_{\mathrm{d}}=-U / 2$. Here $T_{\mathrm{K}}(\Delta)$ stands for $T_{\mathrm{K}}^{\mathrm{HCM}}(\Delta)$ defined in the main text.

\section{Kondo temperature}

The Kondo temperature is the most basic energy scale that characterizes the correlated Kondo state. As remarked already, in the regions $N_{\mathrm{g}} \approx 1$ and $N_{\mathrm{g}} \approx 3$ it is dramatically suppressed for large Kramers pair splittings $\Delta>T_{\mathrm{K}}^{\mathrm{SU}(4)}$. This suppressed $\mathrm{SU}(2)$ Kondo temperature, $T_{\mathrm{K}}^{\mathrm{SU}(2)}$, can be estimated by carrying out a two-step RG procedure [32].

The Kondo temperature has first been defined in the context of a simple spin exchange Hamiltonian of the form $\hat{H}_{\text {int }}=J \mathbf{S} \cdot \mathbf{s}$, describing the interaction of a magnetic moment $\mathbf{S}$ at the origin with the local spin density $\mathbf{s}$ of the conduction electrons, with $J>0$ an antiferromagnetic exchange coupling. Later on, it was realized that the symmetry of the exchange Hamiltonian plays a crucial role and affects the Kondo temperature. The $\mathrm{SU}(N)$-Kondo model [33], in particular, ${ }^{2}$ yields a Kondo temperature $T_{\mathrm{K}}^{\mathrm{SU}(N)} \approx W e^{-2 / N \rho_{0} J}$, implying that a larger $N$ appreciably enhances $T_{\mathrm{K}}$. This behavior can be obtained by carrying out a renormalization group analysis for the effective exchange coupling (vertex function) describing scattering processes at energy $\epsilon$. To leading logarithmic order, the dimensionless effective exchange coupling, $j(\epsilon) \equiv \rho_{0} J_{\text {eff }}$, satisfies the RG equation

$\frac{\mathrm{d} j}{\mathrm{~d} x}=\frac{N}{2} j^{2}$,

with $x=\ln (W / \epsilon)$ the scaling parameter, and diverges logarithmically at the Kondo scale $\epsilon=T_{\mathrm{K}}^{\mathrm{SU}(\mathrm{N})}$. $^{3}$

Let us now focus on the Kondo regime $(\Gamma \ll U)$ of a CNT quantum dot with $N_{\mathrm{g}}=1$ electrons trapped inside a shell. In this regime, assuming further $\Delta \ll U$, the interaction of the electrons with the nanotube can be described by an exchange Hamiltonian of almost perfect $\mathrm{SU}(4)$ symmetry [7]. The structure of the vertex function (effective exchange amplitude), however, depends on the energy of the electrons scattered. Electrons of very high energy, $\epsilon \gg \Delta$, can induce transitions between all four levels, and experience an SU (4) exchange process. Correspondingly their scattering amplitude satisfies (10) with $N=4$. Electrons of energy $\epsilon \ll \Delta$, however, can only flip the states within the lower Kramers doublet, and their scattering amplitude obeys (10) with $N=2$. Matching the $N=2$ and $N=4$ solutions of Eq. (10) at $x=\ln (W / \Delta)$ we thus obtain

$j(\epsilon<\Delta)=\frac{1}{\ln \left[\epsilon \Delta /\left(T_{K}^{\mathrm{SU}(4)}\right)^{2}\right]}$.

The energy where the perturbative RG breaks down can be

\footnotetext{
${ }^{2}$ The $\mathrm{SU}(N)$ Hamiltonian is defined in terms of an $N$-fold degenerate level as $\hat{H}_{\text {int }}=(J / 2) \hat{X}$, with $\hat{X}$ the exchange operator

${ }^{3}$ Both $\epsilon$ and $W$ can be considered as scaling variables here.
}

identified as the Kondo temperature $T_{\mathrm{K}}$, and is expressed as

$T_{\mathrm{K}}(\Delta) \propto \frac{U^{2}}{\Delta} e^{-1 / j}$,

predicting a $\sim 1 / \Delta$ decay of the Kondo temperature for $\Delta<U$. Notice that in this expression, we replaced the bandwidth by $U$, which serves as an upper cutoff for spin exchange processes within the Anderson model.

As we now demonstrate, the analytical expression (11) is in good agreement with our NRG calculations. The definition of $T_{\mathrm{K}}$ is not unique, we have therefore extracted it from the NRG data in two different ways: in addition to the half conductance scale $T_{\mathrm{K}}^{\mathrm{HCM}}(\Delta)$ defined earlier, we also introduced the Kondo scale $T_{\mathrm{K}}^{\mathrm{HWHM}}(\Delta)$ as the half width at half maximum of the spectral function for the $\hat{d}_{j}$ operators. Both Kondo temperatures are shown in Fig. 7, where three regimes can be delineated: for $\Delta<T_{\mathrm{K}}^{\mathrm{SU}(4)}$, the system is governed by $\mathrm{SU}(4)$ physics, and $T_{\mathrm{K}}$ agrees with the $\mathrm{SU}$ (4) Kondo temperature. This is followed by a "crossover region", $T_{\mathrm{K}}^{\mathrm{SU}(4)}<\Delta<U$, where an $\mathrm{SU}(4) \rightarrow \mathrm{SU}(2)$ crossover takes place as a function of temperature or energy, but below $\Delta$ an $\mathrm{SU}(2)$ Kondo state emerges with a suppressed Kondo temperature given by (11). Finally, for $\Delta>U$ only one Kramers doublet remains active, and an $\mathrm{SU}(2)$ Kondo behavior appears at all scales with a $T_{\mathrm{K}}$ independent of $\Delta$.

\section{Transport in a finite magnetic field}

A finite magnetic field turns out to be crucial to disentangle the effects of SOI and valley mixing. As shown below, both the spectral function and the linear conductance display qualitatively different features depending on the direction of the applied magnetic field.

In finite magnetic field the $N_{\mathrm{g}}=1$ (center of the first Coulomb valley) condition becomes $\varepsilon_{\mathrm{d}}=-U / 2-\left(\varepsilon_{4}\left(B_{\|}\right)+\varepsilon_{3}\left(B_{\|}\right)\right) / 2$, whereas $N_{\mathrm{g}}=2$ (particle-hole symmetric point) remains $\varepsilon_{\mathrm{d}}=-3 U / 2$.

\subsection{Parallel magnetic field}

Spectrum. The energy levels of the CNT Hamiltonian, Eq. (1), easily follow from the discussion in Appendix A.2. They read

$\varepsilon_{1,4}=\varepsilon_{\mathrm{d}}+\frac{1}{2} g_{S} B_{\|} \pm \frac{1}{2} \sqrt{\Delta_{\mathrm{KK}^{\prime}}^{2}+\left(\Delta_{\mathrm{SO}}+2 g_{\text {orb }} B_{\|}\right)^{2}}$,

$\varepsilon_{2,3}=\varepsilon_{\mathrm{d}}-\frac{1}{2} g_{\mathrm{s}} B_{\|} \pm \frac{1}{2} \sqrt{\Delta_{\mathrm{KK}^{\prime}}^{2}+\left(\Delta_{\mathrm{SO}}-2 g_{\mathrm{orb}} B_{\|}\right)^{2}}$

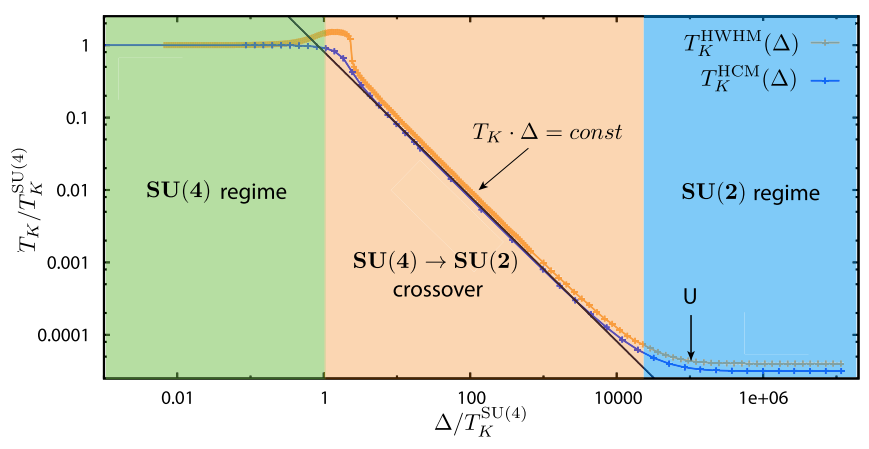

Fig. 7. Evolution of the Kondo temperature as a function of $\Delta$. The Kondo temperature was extracted from the NRG data in two different ways: (i) as the half width at half maximum of the total spectral function (HWHM) and (ii) half conductance maximum (HCM). The other parameters were fixed to $U=W, \varepsilon_{\mathrm{d}}=-U / 2$ $\left(N_{\mathrm{g}}=1\right), \Gamma=U / 50$ and $T=0$. 


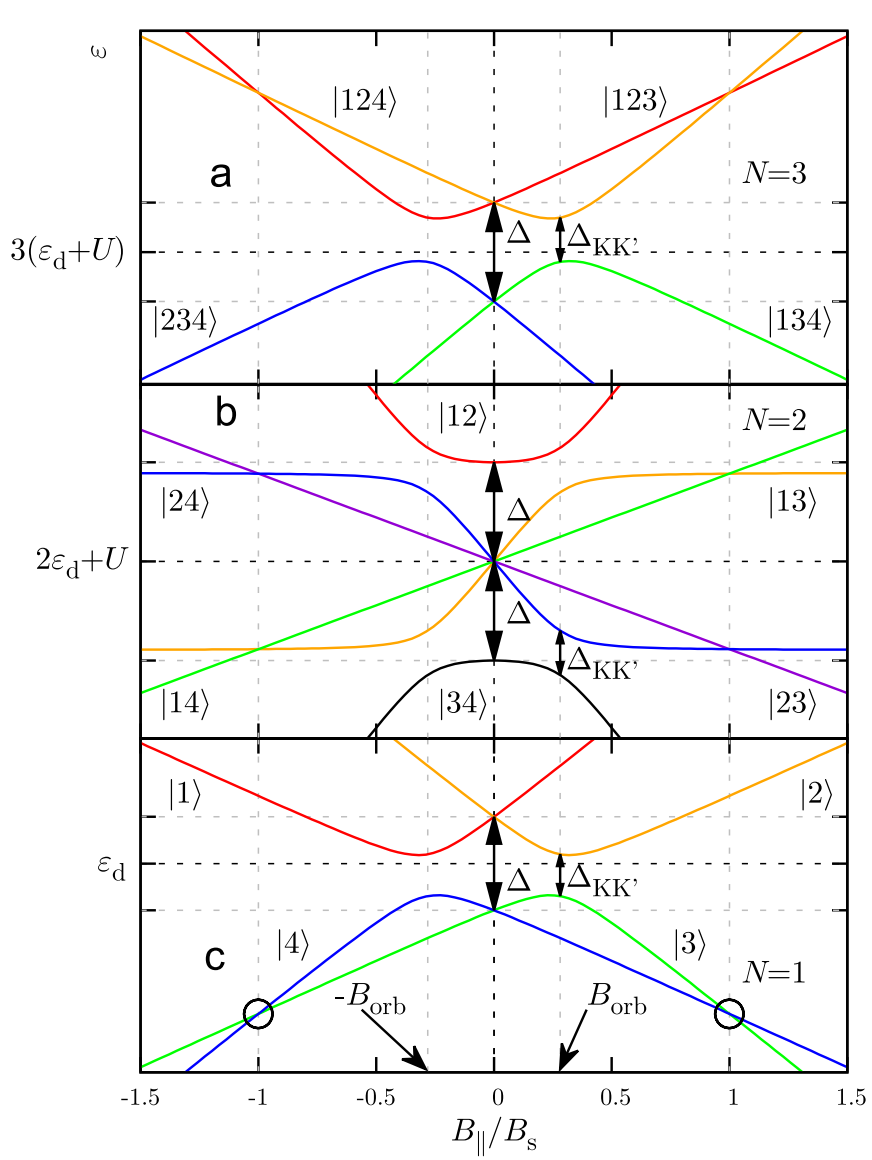

Fig. 8. Parallel magnetic field dependence of the energy levels for (a) $N=3$, (b) $N=2$ and (c) $N=1$ Hilbert space sectors. Black circles indicate ground-state levels crossing. The parameters are $U=W, \Gamma=0.02 W, \varepsilon_{\mathrm{d}}=-U / 2, g_{\mathrm{s}}=2, g_{\text {orb }}=1.83 g_{\mathrm{s}}$ and $\Delta_{\mathrm{KK}^{\prime}}=\Delta \mathrm{so} / 2=5 \Gamma$. They are chosen such that the inequality (13) is fulfilled.

where $g_{s}\left(g_{\text {orb }}\right)$ is the Landé spin(orbital) $g$-factor and $B_{\|}$the amplitude of the parallel magnetic field.

The resulting spectrum for occupancies with $N=1,2,3$ of the CNT is shown in Fig. 8. It is interesting to notice the crossing of the states $|3\rangle$ and $|4\rangle$ in the sector $N=1$ (circles in Fig. 8(c)). It can occur if

$\frac{\left|\Delta_{\mathrm{KK}^{\prime}}\right|}{\left|\Delta_{\mathrm{SO}}\right|}<\sqrt{\frac{4 g_{\mathrm{orb}}^{2}}{g_{\mathrm{s}}^{2}}-1}$,

at a magnetic field value given by

$B_{\mathrm{s}}=\sqrt{\frac{\Delta_{\mathrm{SO}}^{2}}{g_{\mathrm{s}}^{2}}-\frac{\Delta_{\mathrm{KK}^{\prime}}^{2}}{4 g_{\mathrm{orb}}^{2}-g_{\mathrm{s}}^{2}}}$.

Thus, the ground states in the one-particle sector of the Hilbert space switch from $|4\rangle$ to $|3\rangle$ for $B_{\|}>B_{s}$. Moreover, an avoided crossing occurs at $B_{\|}=B_{\text {orb }}=\Delta_{\text {so }} /\left(2 g_{\text {orb }}\right)$. If Eq. (13) is not satisfied, the crossing of the two states in the $N=1$ sector cannot be achieved, as it is shown in Fig. 9.

Focusing on the $N=2$ sector of the Hilbert space (see Fig. 8(b)), one notices that again an avoided crossing occurs at $B_{\text {orb }}=\Delta_{\mathrm{SO}} / 2 g_{\text {orb }}$, which becomes an exact crossing for $\Delta_{\mathrm{KK}^{\prime}}=0$. In this special case the two states $|34\rangle$ and 124$\rangle$ are degenerate. Finally, no ground state crossing is observed for $N=3$ (see Fig. 8(a)).

Using these considerations, we can build up the ground state configuration of the system as a function of the applied parallel magnetic field and of the orbital energy $\varepsilon_{\mathrm{d}}$ (see Fig. 10). By inspecting where a crossing occurs (dashed lines in Fig. 10), it is possible to capture the so-called "Kondo revivals" [17], where the

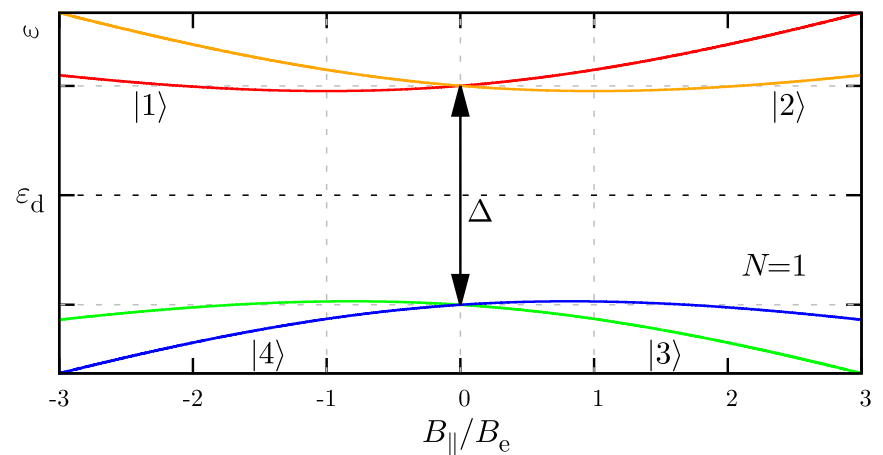

Fig. 9. Evolution of the single particle energy levels in parallel magnetic field. The parameters are $\Gamma=0.02 W, U=W, \varepsilon_{\mathrm{d}}=-U / 2, \Delta \mathrm{so}=5 \Gamma, g_{\mathrm{s}}=2, g_{\text {orb }}=1.83 g_{\mathrm{s}}$ and $\Delta_{\mathrm{KK}}=10 \Delta \mathrm{so} \sqrt{4 g_{\mathrm{orb}}^{2} / g_{\mathrm{s}}^{2}-1}$. For this choice of parameters the inequality (13) is not satisfied and no level crossing is possible at finite magnetic field. Here, $B_{\mathrm{e}}>0$ corresponds to the value of $B_{\|}$such that the state $|2\rangle$ shows a minimum.

Kondo effect is restored for some specific values of the applied magnetic field. As Fig. 10 reveals, revivals are expected around $B_{\|}=B_{\text {orb }}$ for $\Delta_{\mathrm{KK}^{\prime}} \simeq 0$ in the $N=2$ valley, and around $B_{\|}=B_{\mathrm{s}}$, if the inequality (13) is fulfilled, in the $N=1$ valley.

This ground-state analysis was for the first time performed in Ref. [17] (see Sec. IV) where the effects of a finite SOI splitting were investigated. In our work we have also included a finite valley mixing which, as shown in Fig. 9, can preclude a crossing and thus, as demonstrated below, a Kondo revival.

Spectral function. The spectral function is shown for several values of the parallel magnetic field in Fig. 11. In the absence of the magnetic field (black curve) it shows a Kondo peak at the Fermi level and two satellite peaks located at $\omega \simeq \pm \Delta= \pm\left(\varepsilon_{1(2)}(0)-\varepsilon_{3(4)}(0)\right)$. As we switch on the magnetic field, the Kondo peak lowers and then splits into two resonances located at $\omega \simeq \pm\left(\varepsilon_{3}\left(B_{\|}\right)-\varepsilon_{4}\left(B_{\|}\right)\right)$, due to processes representing quantum fluctuations within the lowest Kramers pair. Increasing the magnetic field, the peaks merge again into one at $B_{\|}=B_{\mathrm{s}}$ (violet curve) and the spectral function recovers the unitary value at $\omega=0$ (see inset of Fig. 11). For larger values of $B_{\|}$the Kondo peak splits again. Regarding the satellite peaks, they lower, broaden and shift towards higher energies. We find that at $B_{\|}=B_{\mathrm{s}}$ the leftmost one is due to a resonance in the third and fourth components ( $A_{3}(\omega)=A_{4}(\omega), \varepsilon_{3}=\varepsilon_{4}$ for $B_{\|}=B_{s}$ ) of the spectral function. On the other hand, the rightmost peak has an internal substructure being the sum of a maximum in the first, $A_{1}(\omega)$, and second, $A_{2}(\omega)$, components. Increasing furthermore the magnetic field $\left(B_{\|}>B_{\mathrm{s}}\right)$ the satellite peaks

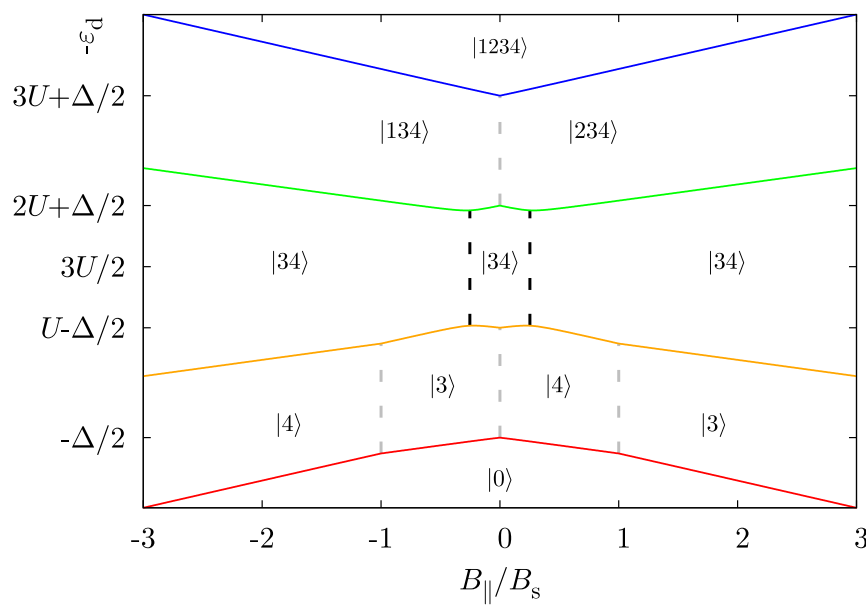

Fig. 10. Ground state configuration as a function of the energy $\varepsilon_{\mathrm{d}}$ and the applied parallel magnetic field. Gray dashed lines indicate degeneracies. The black dashed line indicates $B_{\|}=B_{\text {orb }}$. Parameters are, as in Fig. 8, $U=W, \Gamma=0.02 W, \varepsilon_{\mathrm{d}}=-U / 2$, $g_{\mathrm{s}}=2, g_{\text {orb }}=1.83 g_{\mathrm{s}}$ and $\Delta_{\mathrm{KK}^{\prime}}=\Delta \mathrm{so} / 2=5 \Gamma$. 


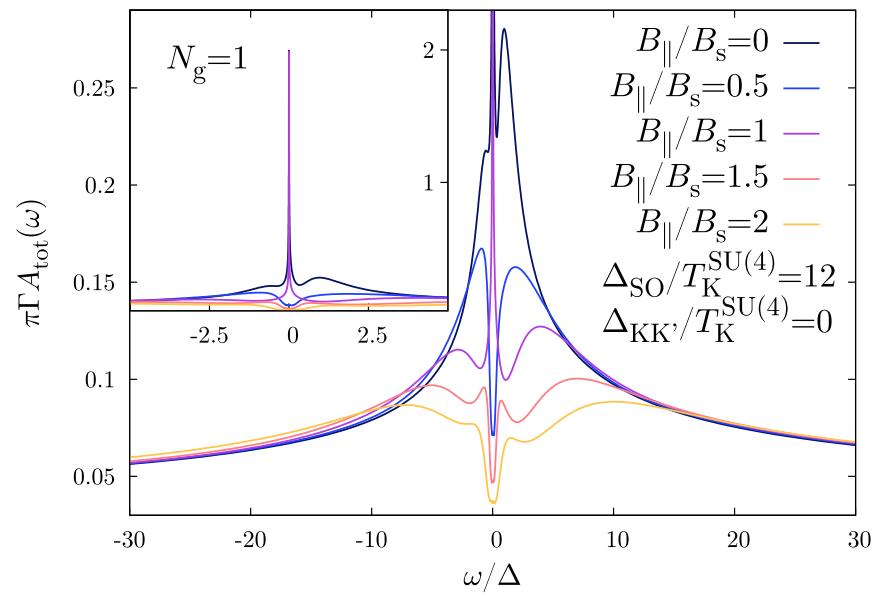

Fig. 11. Close-up image of the spectral function as a function of the frequency for $\Delta_{\mathrm{KK}^{\prime}}=0$ and several values of the parallel magnetic field in the middle of the Coulomb valley with $N_{\mathrm{g}}=1$ at $T=0$. Inset: Full spectral function. The unitary limit is reached for $B_{\|}=0$ while the full Kondo revival is obtained at $B_{\|}=B_{\mathrm{s}}$. (For interpretation of the references to color in this figure caption, the reader is referred to the web version of this paper.)

lower and broaden, the leftmost being determined essentially by the component corresponding to the ground state, $A_{3}(\varepsilon)$. Despite the fact that the energy separation, $\varepsilon_{2}\left(B_{\|}\right)-\varepsilon_{1}\left(B_{\|}\right)$, of the excited states $|1\rangle$ and $|2\rangle$ is monotonically increasing, the total spectral function does not show a split of the corresponding resonances in this range of investigated parallel magnetic field values $\left(0 \leq B_{\|} \leq 2 B_{s}\right.$ with $B_{\mathrm{S}} \simeq 90 T_{\mathrm{K}}(\Delta)$ ). Our results on the impact of a parallel magnetic field on the spectral function of a CNT are consistent with the NRG analysis performed in Ref. [8] when $\Delta_{\mathrm{SO}}=\Delta_{\mathrm{KK}^{\prime}}=0$.

Linear conductance. In Fig. 12 we show the linear conductance of the system as a function of the applied parallel magnetic field and for a fixed ratio of $\Delta / T_{\mathrm{K}}^{\mathrm{SU}(4)} \simeq 12$. In Fig. 12(a) the conductance shows, as expected, the Kondo revival at $B_{\|}=B_{s}$. Moreover, the width of the Kondo peak is proportional to $T_{\mathrm{K}}(\Delta)$. By increasing the valley mixing term $\Delta_{\mathrm{KK}^{\prime}}$ the resonance shifts towards $B_{\|}=0$ and, in case $\Delta_{\mathrm{KK}^{\prime}} / \Delta_{\mathrm{SO}}$ does not fulfill Eq. (13), the Kondo revival disappears (black solid line).

In the $N_{\mathrm{g}}=2$ valley, Fig. 12(b), the valley mixing term acts slightly differently. For $\Delta_{\mathrm{KK}^{\prime}}=0$ the SU(4)- Kondo effect in $B_{\|}=0$ is essentially suppressed for this set of parameters $\left(\Delta / T_{\mathrm{K}}^{\mathrm{SU}(4)} \simeq 12\right.$ ) and hence a Kondo peak is present only at $B_{\|}=B_{\text {orb }}$. Switching on the valley mixing, since $B_{\text {orb }}$ is not $\Delta_{\mathrm{KK}^{\prime}}$ dependent, results in the suppression of this resonance without any shift. We notice that Kondo revivals have indeed been seen in experiments on CNT-dots in parallel fields $[9,15]$.

\subsection{Perpendicular magnetic field}

Spectrum. A magnetic filed perpendicular to the CNT axis couples only to the spin degree of freedom. As it follows from A.3, the energy levels of the CNT Hamiltonian (1) in the presence of a perpendicular magnetic field read

$\varepsilon_{1,4}=\varepsilon_{\mathrm{d}} \pm \frac{1}{2} \sqrt{\Delta_{\mathrm{SO}}^{2}+\left(\Delta_{\mathrm{KK}^{\prime}}+g_{\mathrm{s}} B_{\perp}\right)^{2}}$,

$\varepsilon_{2,3}=\varepsilon_{\mathrm{d}} \pm \frac{1}{2} \sqrt{\Delta_{\mathrm{SO}}^{2}+\left(\Delta_{\mathrm{KK}^{\prime}}-g_{\mathrm{s}} B_{\perp}\right)^{2}}$,

where $B_{\perp}$ is the amplitude of the perpendicular magnetic field. The corresponding spectrum is shown in Fig. 13. It shows clear qualitative differences with respect to the parallel case. For example, no ground state crossing is observed for $N=1$ and $N=3$ : the distance

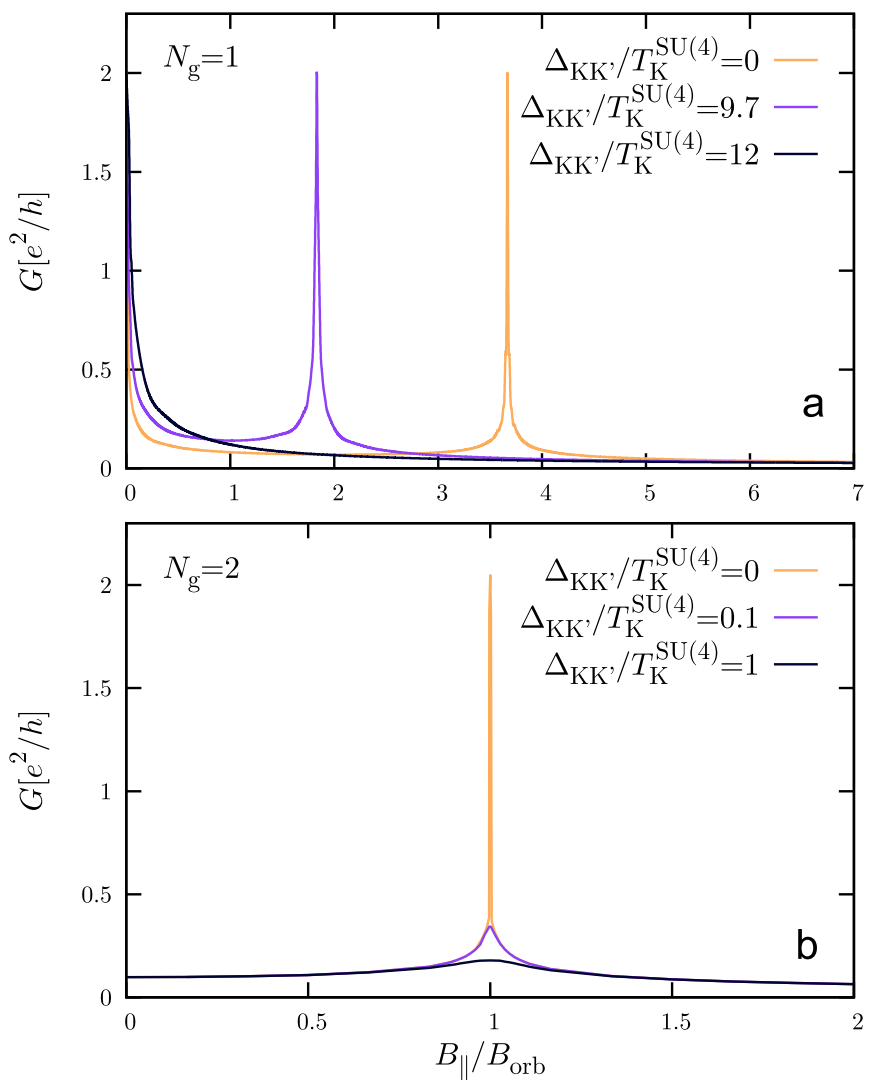

Fig. 12. Linear conductance as a function of the parallel magnetic field (a) in the middle of the Coulomb valley, $N_{\mathrm{g}}=1$ and (b) at the particle-hole symmetric point $N_{\mathrm{g}}=2$ at $T=0 . \Delta \mathrm{So}$ and $\Delta_{\mathrm{KK}^{\prime}}$ were varied such that $\Delta / T_{\mathrm{K}}^{\mathrm{SU}(4)}$ remained constant.

between the lowest energy levels first increases and then saturates to the valley mixing strength $\Delta_{\mathrm{KK}^{\prime}}$. An avoided crossing between excited states occurs for $B_{\perp}= \pm B_{\mathrm{KK}^{\prime}}$, where $B_{\mathrm{KK}^{\prime}}=\Delta_{\mathrm{KK}^{\prime}} / g_{\mathrm{s}}$. On the other hand, in the $N=2$ sector, the avoided crossing at $B_{\perp}= \pm B_{\mathrm{KK}^{\prime}}$ is between the ground state $|34\rangle$ and the state |24〉. Hence an exact crossing occurs for $\Delta_{\text {so }}=0$. Comparing Figs. 13 and 8 we indeed see that the SOI and the valley mixing exchange their role changing from parallel to perpendicular magnetic fields. Following the same argument of the previous section, this revels that the Kondo revival can be achieved only in the $N_{\mathrm{g}}=2$ valley for $B_{\perp}=B_{\mathrm{KK}}$.

Spectral function. We analyze the behavior of the total spectral function at $N_{g}=1$ for several values of the perpendicular magnetic field in Fig. 14. Since the Kondo revival is not possible in the first valley, the Kondo peak monotonically splits into two sub-peaks located at $\omega= \pm\left(\varepsilon_{3}\left(B_{\perp}\right)-\varepsilon_{4}\left(B_{\perp}\right)\right)$. Furthermore, two satellite peaks are visible. The rightmost resonance is the sum of two peaks in $A_{1}(\varepsilon)$ and $A_{2}(\varepsilon)$, whereas the leftmost one is essentially determined by the fourth component, $A_{4}(\varepsilon)$, corresponding to the ground state level. As in the parallel case, no splitting of the satellites is observed in the investigated magnetic field range. For $B_{\perp} \geq B_{\mathrm{KK}^{\prime}} / 2$ the central Kondo peaks merge with the lateral satellites.

Linear conductance. In Fig. 15 we show the linear conductance as a function of the applied perpendicular magnetic field. A perfect Kondo revival is obtained only if $\Delta_{\mathrm{SO}}=0$; for $\Delta_{\mathrm{SO}} \neq 0$ the Kondo resonance is suppressed because the degeneracy between the states $|34\rangle$ and $|24\rangle$ is lifted and the fluctuations of the quantum numbers referring to these states become energetically unfavorable.

\section{Conclusion}

In this work, we have investigated the transport and spectral 


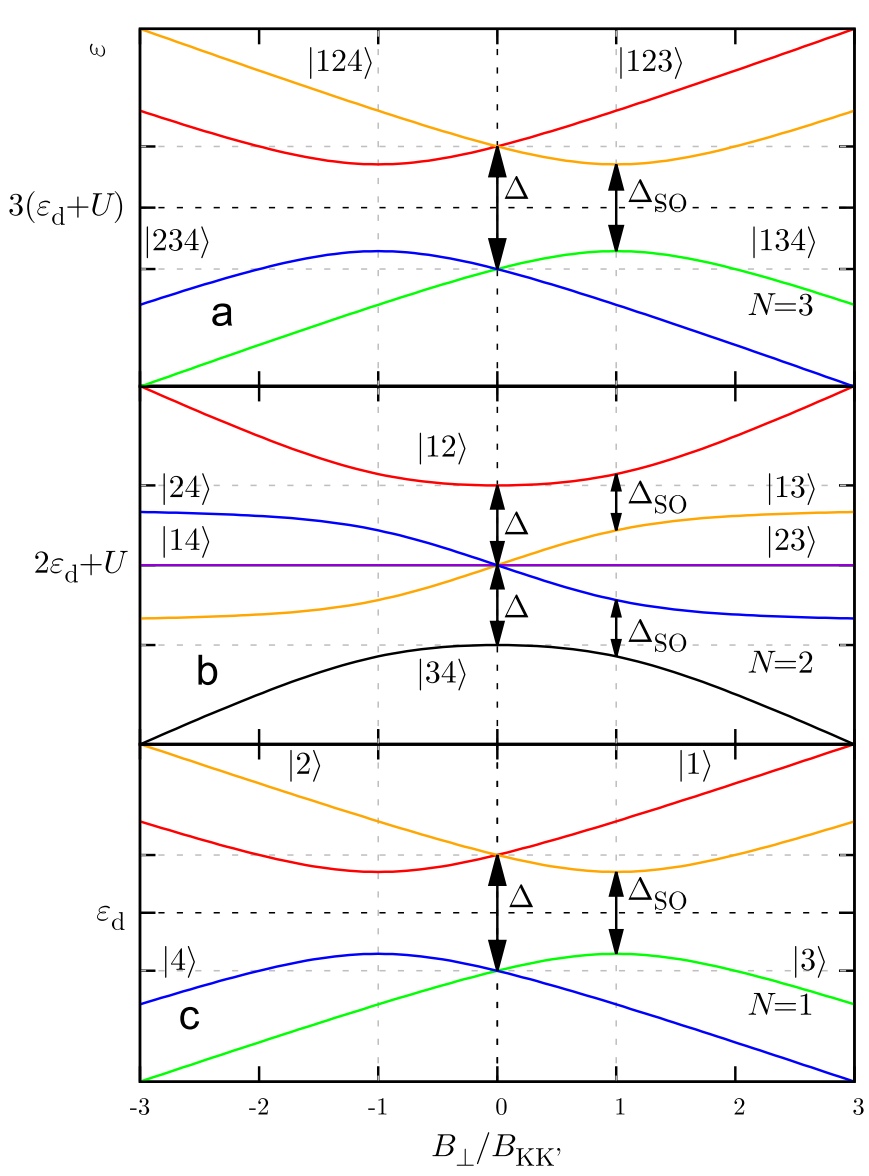

Fig. 13. Perpendicular magnetic field dependence of the energy levels for (a) $N=3$, (b) $N=2$ and (c) $N=1$ particle number.

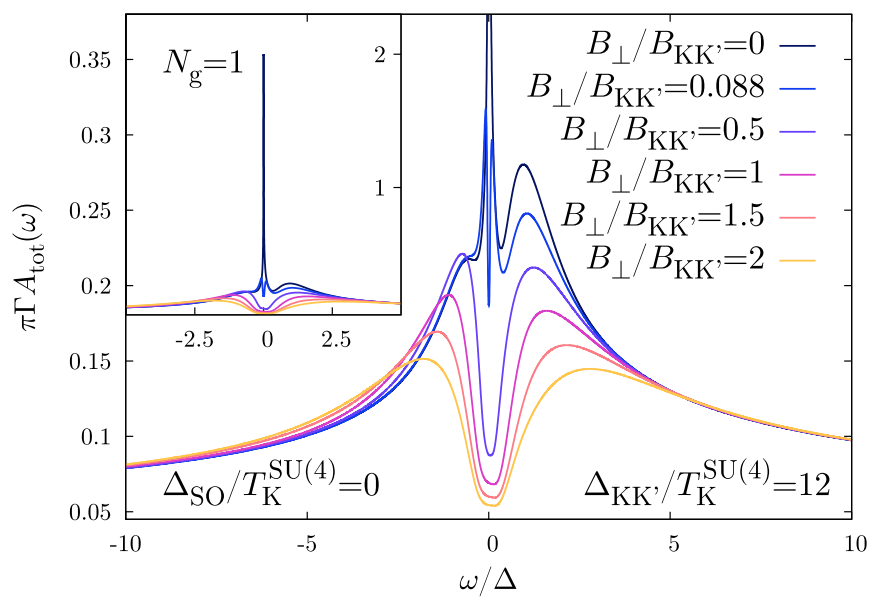

Fig. 14. Close-up structure of the total spectral function as a function of the frequency for several values of the perpendicular magnetic field, for $N_{g}=1$ and $T=0$. Inset: Full spectral function, showing that the unitary limit is only obtained for $B_{\perp}=0$.

properties of CNTs in the Kondo regime. To do that, we have constructed a model that, for the sake of simplicity, assumes spin and valley quantum number conservation during tunneling processes, but accounts for the combined effects of spin-orbit interaction, valley mixing, and electron-electron interaction, $U$, and finite magnetic field.

First, we carried out a detailed study of the cross-over between the $\mathrm{SU}(4)$ and $\mathrm{SU}(2)$ - Kondo regime by analyzing the dependence of the spectral functions and the linear conductance on the Kramers pair splitting, $\Delta$. We have shown by means of DM-NRG

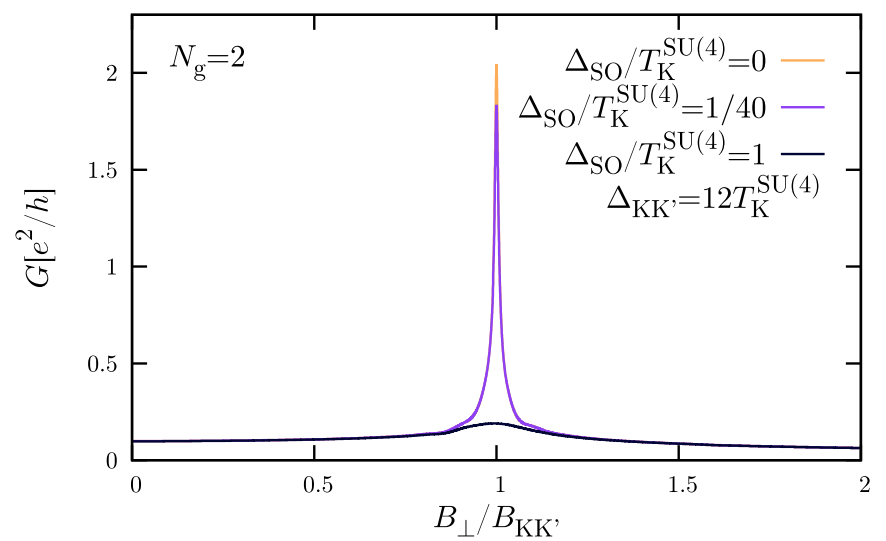

Fig. 15. Linear conductance as a function of the perpendicular magnetic field at the particle-hole symmetric point, $N_{\mathrm{g}}=2$ at $T=0$.

computations, in particular, that in the Kondo regime of a singly occupied CNT longitudinal mode a universal SU(4) conductance is displayed for $\Delta<T_{\mathrm{K}}^{\mathrm{SU}(4)}$, while for $\Delta \gg T_{\mathrm{K}}^{\mathrm{SU}(4)}$ the conductance and the spectral functions display characteristic $\mathrm{SU}(2)$ behavior with a strongly reduced Kondo temperature. In the intermediate regime $T_{\mathrm{K}}^{\mathrm{SU}(4)} \ll \Delta \ll U$ we observed a $1 / \Delta$ dependence of the Kondo temperature. This behavior can be explained in terms of simple scaling arguments, and is also in agreement with exact Bethe Ansatz results obtained in case of infinitely strong Coulomb repulsion [34,28].

Finally, we analyzed the magnetic field dependence of the linear conductance and the spectral function, both in fields parallel and perpendicular to the CNT axis. Along the lines of Ref. [17], we derived the necessary conditions to observe the Kondo revival, while extending our analysis to finite valley mixing values $\left(\Delta_{\mathrm{KK}^{\prime}} \neq 0\right)$ and to the case of perpendicular magnetic fields. We showed that in both parallel and perpendicular fields, the total spectral function of the system shows a four peak structure (two for the absorption and two for the emission processes). We showed that in the case of odd occupancy of a CNT shell, a large enough $\Delta_{\mathrm{KK}^{\prime}}$ can prevent the occurrence of a Kondo revival in parallel field; in perpendicular fields no revival is expected. Kondo revivals have indeed so far been observed experimentally only for parallel magnetic fields $[15,18]$. In case of a perpendicular magnetic field, the outer peaks can merge with those of the split Kondo resonance, thereby leading to a two-peak structure (one for absorption and one for emission processes) for large magnetic field values compared to the $\mathrm{SU}(4)$ - Kondo temperature and $B_{\perp}>B_{\mathrm{KK}^{\prime}} / 2$.

\section{Acknowledgments}

We gratefully thank Magdalena Marganska and Sergey Smirnov for the fruitful discussions. We acknowledge financial support through DFG Program no. GRK1570 and the Hungarian Research Grant no. OTKA K105149. Part of this work has been completed at the Aspen Center for Physics, supported by National Science Foundation Grant PHY-1066293.

\section{Appendix A. Orthogonal transformations}

In this appendix we present the construction of the Hamiltonian introduced in Eq. (1) in the Kramers basis from an underlying Anderson model that takes into account the carbon nanotube structure $[13,18]$. In the absence of the valley mixing and spin- 
orbit interaction, the basis set $\left\{\left|K^{\prime}, \uparrow\right\rangle,\left|K^{\prime}, \downarrow\right\rangle,|K, \uparrow\rangle,|K, \downarrow\rangle\right\}$, indexed by the valley and spin quantum numbers, is orthogonal. When $\Delta \neq 0$ this is no longer true, and it is suitable to adopt instead the bonding (anti-bonding) representation $\{|a, \uparrow\rangle,|b, \uparrow\rangle,|a, \downarrow\rangle,|b, \downarrow\rangle\}$, which can be constructed as

$|a, \sigma\rangle=\hat{d}_{+, \sigma}^{\dagger}|0\rangle=\frac{\left|K^{\prime}, \sigma\right\rangle+|K, \sigma\rangle}{\sqrt{2}}$,

$|b, \sigma\rangle=\hat{d}_{-, \sigma}^{\dagger}|0\rangle=\frac{\left|K^{\prime}, \sigma\right\rangle-|K, \sigma\rangle}{\sqrt{2}}$.

In this basis the CNT Hamiltonian consists of several terms

$\hat{H}_{\mathrm{CNT}}=\hat{H}_{\mathrm{CNT}}^{(0)}+\hat{H}_{e-e}+\hat{H}_{\mathrm{B}}=\hat{H}_{\mathrm{d}}+\hat{H}_{\mathrm{KK}^{\prime}}+\hat{H}_{\mathrm{SO}}+\hat{H}_{e-e}+\hat{H}_{\mathrm{B}}$,

where $\hat{H}_{\mathrm{d}}$ is the $\mathrm{SU}(4)$ invariant component and $\varepsilon_{\mathrm{d}}$ the orbital energy which can be tuned through the applied gate voltage. Explicitly,

$\hat{H}_{\mathrm{d}}=\varepsilon_{\mathrm{d}} \sum_{i, \sigma= \pm} \hat{d}_{i, \sigma}^{\dagger} \hat{d}_{i, \sigma}$.

We add to the pure SU(4) term, respectively, the valley mixing (Eq. (A.4a)) and the SOI (Eq. (A.4b)) components:

$\hat{H}_{\mathrm{KK}^{\prime}}=\frac{\Delta_{\mathrm{KK}^{\prime}}}{2} \sum_{i, \sigma= \pm} i \hat{d}_{i, \sigma}^{\dagger} \hat{d}_{i, \sigma}$

$\hat{H}_{\mathrm{SO}}=\frac{\Delta_{\mathrm{SO}}}{2} \sum_{i, \sigma= \pm} \sigma \hat{d}_{-i, \sigma}^{\dagger} \hat{d}_{i, \sigma}$.

Notice that in the bonding/anti-bonding basis the valley mixing effect translates in an energy difference between the bonding and anti-bonding states [13]. On the other hand, the SOI (due to curvature effects and relativistic correction to the CNT Hamiltonian) is off-diagonal.

The fourth term in Eq. (A.2) describes the electron-electron interaction with $U$ associated to the charging energy of the dot

$\hat{H}_{e-e}=\frac{U}{2} \sum_{i \neq i_{1}= \pm} \sum_{\sigma, \sigma 1= \pm} \hat{d}_{i, \sigma}^{\dagger} \hat{d}_{i, \sigma} \hat{d}_{i_{1}, \sigma_{1}}^{\dagger} \hat{d}_{i_{1}, \sigma}$.

The external magnetic field enters through the last term in Eq. (A.2)).

\section{A.1. Spectrum for zero magnetic field}

The single particle Hamiltonian equation (A.2) can be diagonalized by a unitary transformation, and the new orthogonal basis is the Kramers basis $\{|1\rangle,|4\rangle,|2\rangle,|3\rangle\}$ used throughout of the present work. The unitary operator is

$\mathcal{U}=\left(\begin{array}{cccc}\cos (\theta) & \sin (\theta) & 0 & 0 \\ -\sin (\theta) & \cos (\theta) & 0 & 0 \\ 0 & 0 & \cos (\theta) & -\sin (\theta) \\ 0 & 0 & \sin (\theta) & \cos (\theta)\end{array}\right)$,

where the angle $\theta$ is given by $\tan (2 \theta)=\Delta_{\mathrm{SO}} / \Delta_{\mathrm{KK}^{\prime}}$. In the diagonalized form, $\hat{H}_{\mathrm{CNT}}$ becomes the dot Hamiltonian from Eq. (1).

We immediately notice that, because the two blocks in Eq. (A.6) yield the same eigenvalues, two pairs of degenerate doublets arise. These are the so-called Kramer pairs that, in our notation, are the couples of states $(1,2)$ and $(3,4)$. As such, the states within each Kramers pair are related through the time reversal operator $\hat{\mathcal{T}}$ as it is sketched in Fig. 2. Additionally, valley reversal, governed by the anti-unitary operator $\hat{\mathcal{P}}$, relates the couples $(1,4)$ and $(2,3)$ originating from the two sub-blocks. In the Kramers basis $\hat{\mathcal{T}}$ and $\hat{\mathcal{P}}$ are given by

$\hat{\mathcal{T}}=\kappa\left(\hat{d}_{2}^{\dagger} \hat{d}_{1}+\hat{d}_{4}^{\dagger} \hat{d}_{3}-\right.$ h. c. $)$

$\hat{\mathcal{P}}=\kappa\left(\hat{d}_{4}^{\dagger} \hat{d}_{1}-\hat{d}_{3}^{\dagger} \hat{d}_{2}-\right.$ h. c. $)$

where $\kappa$ stands for the complex conjugation. Finally, defining $\hat{C}=\hat{\mathcal{P}} \cdot \hat{\mathcal{T}}^{-1}$, it is possible to relate the couples $(1,3)$ and $(2,4)$ to each other through the unitary operator

$\hat{C}=\hat{d}_{1}^{\dagger} \hat{d}_{3}+\hat{d}_{2}^{\dagger} \hat{d}_{4}+$ h. c.

\section{A.2. Spectrum for finite parallel magnetic field}

An external magnetic field parallel to the CNT axis couples to both the spin degree of freedom and the "orbital" one. Thus, in the bonding(anti-bonding) basis, $\hat{H}_{\mathrm{B}}$ reads [18]

$\hat{H}_{\mathrm{B}}=\hat{H}_{\mathrm{B}-\mathrm{s}}^{\|}+\hat{H}_{\mathrm{B}-\mathrm{orb}}^{\|}=\frac{1}{2} g_{\mathrm{s}} B_{\|} \sum_{i, \sigma= \pm} \sigma \hat{d}_{i, \sigma}^{\dagger} \hat{d}_{i, \sigma}+g_{\text {orb }} B_{\|} \sum_{i, \sigma= \pm} \hat{d}_{-i, \sigma}^{\dagger} \hat{d}_{i, \sigma}$

where $B_{\|}$is the amplitude of the parallel magnetic field. Thus, the diagonalized single particle Hamiltonian is

$\mathcal{U}^{\dagger}\left(B_{\|}\right)\left(\hat{H}_{\mathrm{CNT}}^{(0)}+\hat{H}_{\mathrm{B}}\right) \mathcal{U}\left(B_{\|}\right)=\sum_{j=1}^{4} \varepsilon_{j} \hat{d}_{j}^{\dagger} \hat{d}_{j}$

where

$\mathcal{U}\left(B_{\|}\right)=\left(\begin{array}{cccc}\cos \left(\theta^{+}\right) & \sin \left(\theta^{+}\right) & 0 & 0 \\ -\sin \left(\theta^{+}\right) & \cos \left(\theta^{+}\right) & 0 & 0 \\ 0 & 0 & \cos \left(\theta^{-}\right) & -\sin \left(\theta^{-}\right) \\ 0 & 0 & \sin \left(\theta^{-}\right) & \cos \left(\theta^{-}\right)\end{array}\right)$

Here we defined $\theta^{ \pm}$in such a way that $\tan \left(2 \theta^{ \pm}\right)=\left(\Delta_{\mathrm{SO}} \pm 2 g_{\text {orb }} B_{\|}\right) / \Delta_{\mathrm{KK}^{\prime}}$. Notice that $\mathcal{U}\left(B_{\|}\right)$posses a similar block structure as in Eq. (A.6). However, due to $\theta^{+} \neq \theta^{-}$, time reversal symmetry is broken.

\section{A.3. Spectrum for finite perpendicular magnetic field}

An external magnetic field perpendicular to the CNT axis couples, differently to the parallel case, only to the spin degree of freedom $[13,18]$. Its action reads

$\hat{H}_{\mathrm{B}}=\frac{1}{2} g_{\mathrm{s}} B_{\perp} \sum_{i, \sigma= \pm} \hat{d}_{i, \bar{\sigma}}^{\dagger} \hat{d}_{i, \sigma}$

The transformation that diagonalizes the single particle Hamiltonian can be decomposed as a product of two orthogonal matrices: 
$\mathcal{U}\left(B_{\perp}\right)=O_{1}\left(B_{\perp}\right) O_{2}\left(B_{\perp}\right)$

$O_{1}\left(B_{\perp}\right)=\left(\begin{array}{cccc}\cos \left(\theta^{+}\right) & \sin \left(\theta^{+}\right) & 0 & 0 \\ -\sin \left(\theta^{+}\right) & \cos \left(\theta^{+}\right) & 0 & 0 \\ 0 & 0 & \cos \left(\theta^{-}\right) & \sin \left(\theta^{-}\right) \\ 0 & 0 & -\sin \left(\theta^{-}\right) & \cos \left(\theta^{-}\right)\end{array}\right)$,

$O_{2}\left(B_{\perp}\right)=\frac{1}{\sqrt{2}}\left(\begin{array}{cccc}1 & 0 & 1 & 0 \\ 0 & 1 & 0 & -1 \\ 1 & 0 & -1 & 0 \\ 0 & 1 & 0 & 1\end{array}\right)$,

where $\theta^{ \pm}$is such that $\tan \left(2 \theta^{ \pm}\right)=\Delta_{\mathrm{So}} /\left(\Delta_{\mathrm{KK}^{\prime}} \pm g_{\mathrm{s}} B_{\perp}\right)$. We notice that $\mathcal{U}\left(B_{\perp}\right)$ mixes both the spin and the bonding(anti-bonding) degrees of freedom, whereas, in the previous case, $\mathcal{U}\left(B_{\|}\right)$was mixing only the latter one.

\section{Appendix B. Universality and the fixed points}

We devote this appendix to a brief analysis of the properties of the SU(2) and SU(4) limiting cases in the $N_{g}=1$ valley. When $\Delta=0$ we expect the system to be at the SU(4) fixed point. In Fig. B1 we compare on one side, the conductance as computed with the help of Eq. (8) for $\Delta=0$, with the universal curve for $G\left(T / T_{K}\right)$ as obtained from an underlying Kondo model with SU(4) symmetry, and we find a perfect agreement. Increasing $\Delta$, the system flows away from the $\mathrm{SU}(4)$ and towards the $\mathrm{SU}(2)$ fixed point. As the other curve in Fig. B1 shows, for large enough $\Delta$ 's, i.e. $\Delta \gg W$, the system has already reached the $\mathrm{SU}(2)$ fixed point.

Recently, in Ref. [10] a heuristical analytical expression was proposed for the universal conductance, of the form

$\frac{G\left(T / T_{\mathrm{K}}\right)}{G_{0}}=\left(1+\left(2^{1 / s}-1\right) \cdot\left(\frac{T}{T_{\mathrm{K}}}\right)^{n}\right)^{-s}$,

in order to reproduce the leading-order in the temperature expansion predicted by the conformal field theory $[35,36]$ for the $\mathrm{SU}(4)$ - Kondo Hamiltonian. In this case the leading order is predicted to be cubic, $G\left(T / T_{\mathrm{K}}\right) / G_{0} \simeq 1-\alpha \cdot\left(T / T_{\mathrm{K}}\right)^{3}+\ldots$, with $\alpha$ a constant of the order $\sim 1$, even though the system has still a Fermi liquid character. This sets $n=3$ in Eq. (B.1) in contrast to the case $n=2$ for the $\mathrm{SU}(2)$ symmetry.

In Fig. B2 we compare the conductance with this heuristic curve for different gate voltages. From the fits of the DM-NRG data (Fig. B2), in the range $0 \leq T / T_{\mathrm{K}} \leq 1$, we obtained a good estimate for $s=0.202 \pm 0.002$ in agreement with Ref. [10]. The heuristic curve reproduces very well the DM-NRG results in a wide range of temperatures, $0 \leq T / T_{K} \leq 10$, and deviations become visible only for large enough temperatures.

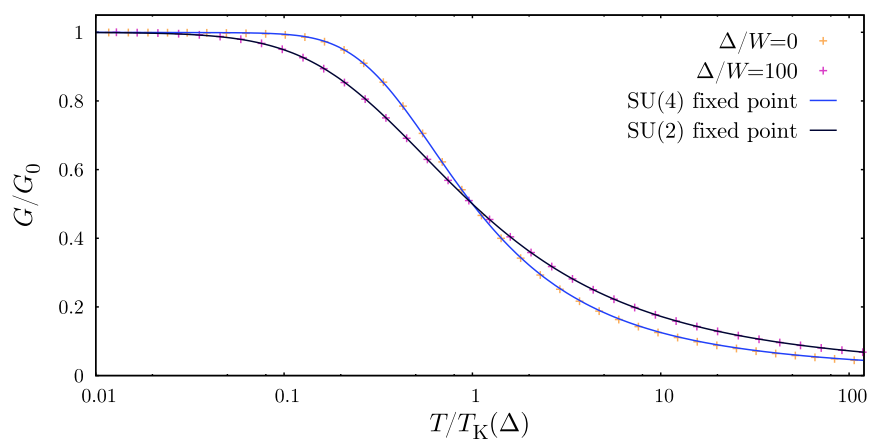

Fig. B1. Comparison between the temperature dependence of the conductance in the limits when $\Delta \rightarrow 0$ and $\Delta \rightarrow \infty$ (symbols) and the SU(2) and SU(4) universal curves for the conductance (solid lines).

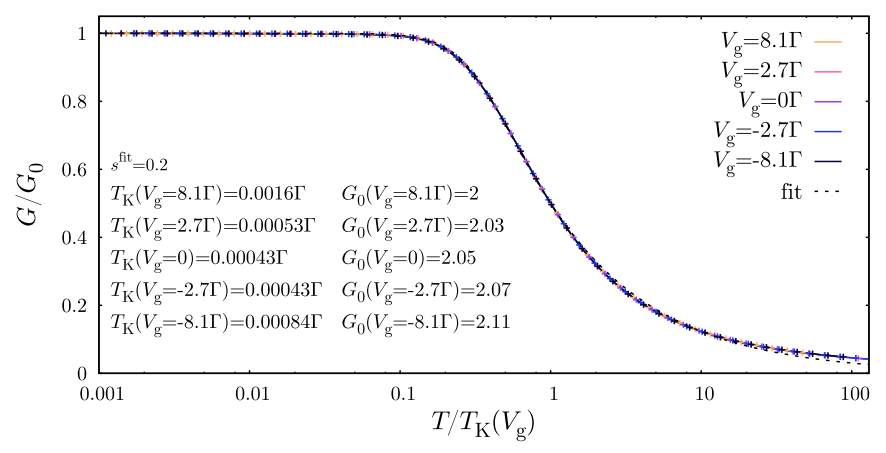

Fig. B2. Universal scaling of the conductance as a function of $T / T_{\mathrm{K}}$ for different gate voltages and comparison with the heuristical curve from Eq. (B.1) with $n=3$. The other parameters are $U=W, \Gamma=U / 50, T=0, \varepsilon_{\mathrm{d}}=-U / 2$ and $\Delta=0$. Here $G_{0}$ is the unitary conductance in unit $e^{2} / h$.

Trying to fit the data for $\Delta \gg W$ in Fig. B1 with $n=2$ we found $s=0.215 \pm 0.002$, in agreement with the predictions from the conformal field theory.

\section{Appendix C. Numerical renormalization group approach}

We solve the Hamiltonian (4) using the numerical renormalization group approach. The core of the NRG is a logarithmic discretization of the conduction band with a parameter $\Lambda \simeq 2$, followed by a mapping of the Hamiltonian to a semi-infinite chain. In this way the problem can be solved perturbatively, as the hopping couplings along the chain, $t_{n}^{j} \sim \Lambda^{-n / 2}$ decrease exponentially [27]. The conduction band Hamiltonian becomes

$\hat{H}_{\text {chain }}=\sum_{j=1}^{4} \sum_{n=0}^{+\infty} t_{n}^{j} \hat{f}_{j, n}^{\dagger} \hat{f}_{j, n+1}+$ h. c..

Here $\hat{f}_{j, n}^{\dagger}$ are the fermionic creation operators at the $n$-th site in the $j$-th channel. The impurity is sitting at site -1 and is coupled to the site $n=0$

$\hat{H}_{\text {tun }}=V \sum_{j=1}^{4} \hat{f}_{j, 0}^{\dagger} \hat{d}_{j}+$ h. c.

As the dot Hamiltonian (1) is not modified by this procedure, the total Hamiltonian consists now of four spinless conduction bands coupled to a complex impurity composed of the dot degrees of freedom.

Along the NRG procedure it is crucial to use the symmetries of the system in order to achieve numerically reliable results. When $\Delta=0$, the model is $\mathrm{SU}(4)$ invariant, as the total Hamiltonian commutes with the SU(4)-spin operator

$\hat{\mathbf{J}}^{\mathrm{SU}(4)}=\frac{1}{2} \sum_{n=-1}^{+\infty} \sum_{j, j^{\prime}=1}^{4} \hat{f}_{j, n}^{\dagger} \lambda_{j j^{\prime}} \hat{f}_{j^{\prime}, n}$,

where $\lambda=\left(\lambda_{1}, \ldots, \lambda_{15}\right)$ is a set of matrices defining the generators for the SU(4) algebra (generalized Gell Mann matrices [37] for example). As discussed in Section 2.2, there are also two $U$ (1) symmetries corresponding to the conservation of charge in each of the Kramers channels. In the NRG language the generators are

$\hat{Q}_{\kappa}=\frac{1}{2} \sum_{j \in \kappa} \sum_{n=-1}^{+\infty}\left(\hat{f}_{j, n}^{\dagger} \hat{f}_{j, n}-\frac{1}{2}\right)$.

Since the generators for spin and for the charges commute among themselves, the system has a global $U(1) \otimes U(1) \otimes S U(4)$ symmetry. The spin orbit and valley mixing perturbations, i.e. $\Delta \neq 0$, break the global symmetry down to the 
$U(1) \otimes U(1) \otimes S U(2) \otimes S U(2)$, generated by the charge and the SU (2)-spin operators

$\hat{\mathbf{J}}_{\kappa}=\frac{1}{2} \sum_{n=-1}^{+\infty} \sum_{j, j^{\prime} \in \kappa} \hat{f}_{j, n}^{\dagger} \boldsymbol{\sigma}_{j j} \hat{f}_{j^{\prime}, n}$,

acting on the two Kramers doublets. Here $\sigma=\left(\sigma_{x}, \sigma_{y}, \sigma_{z}\right)$ is the vector of Pauli matrices.

\section{References}

[1] A.C. Hewson, The Kondo Problem to Heavy Fermions, Cambridge University Press, Cambridge, 1997

[2] E.A. Laird, F. Kuemmeth, G. A. Steele, K. Grove-Rasmussen, J. Nygård, K. Flensberg, L.P. Kouwenhoven, Quantum transport in carbon nanotubes, Rev. Mod. Phys. American Physical Society, 87(3), 2015, 703-764, numpages $=\{62\}$, 10.1103/RevModPhys.87.703, url = \{http://link.aps.org/doi/10.1103/ RevModPhys.87.703\}.

[3] L. Borda, G. Zaránd, W. Hofstetter, B.I. Halperin, J. von Delft, SU(4) Fermi liquid state and spin filtering in a double quantum dot system, Phys. Rev. Lett. 90 (2003) 026602, http://dx.doi.org/10.1103/PhysRevLett.90.026602, URL 〈http:// link.aps.org/doi/10.1103/PhysRevLett.90.026602).

[4] G. Zaránd, A. Brataas, D. Goldhaber-Gordon, Kondo effect and spin filtering in triangular artificial atoms, Solid State Commun. 126 (2003) 463, http://dx.doi. org/10.1016/S0038-1098(03)00180-7, URL 〈http://dx.doi.org/10.1016/S00381098(03)00180-7〉.

[5] S. Sasaki, S. Amaha, N. Asakawa, M. Eto, S. Tarucha, Enhanced Kondo effect via tuned orbital degeneracy in a spin $1 / 2$ artificial atom, Phys. Rev. Lett. 93 (2004) 017205, http://dx.doi.org/10.1103/PhysRevLett.93.017205, URL 〈http://link.aps. org/doi/10.1103/PhysRevLett.93.017205〉.

[6] P. Jarillo-Herrero, J. Kong, H.S.J. van der Zant, C. Dekker, L.P. Kouwenhoven, S. De Franceschi, Orbital Kondo effect in carbon nanotubes, Nature 434 (7032) (2005) 484-488, http://dx.doi.org/10.1038/nature03422, URL 〈http://dx.doi. org/10.1038/nature03422).

[7] M.-S. Choi, R. López, R. Aguado, SU(4) Kondo effect in carbon nanotubes, Phys. Rev. Lett. 95 (2005) 067204, http://dx.doi.org/10.1103/PhysRevLett.95.067204, URL 〈http://link.aps.org/doi/10.1103/PhysRevLett.95.067204〉.

[8] J.S. Lim, M.-S. Choi, M.Y. Choi, R. López, R. Aguado, Kondo effects in carbon nanotubes: from SU(4) to SU(2) symmetry, Phys. Rev. B 74 (2006) 205119, http://dx.doi.org/10.1103/PhysRevB.74.205119, URL 〈http://link.aps.org/doi/10. 1103/PhysRevB.74.205119).

[9] P. Jarillo-Herrero, J. Kong, H.S.J. van der Zant, C. Dekker, L.P. Kouwenhoven, S. De Franceschi, Electronic transport spectroscopy of carbon nanotubes in a magnetic field, Phys. Rev. Lett. 94 (2005) 156802, http://dx.doi.org/10.1103/ PhysRevLett.94.156802, URL 〈http://link.aps.org/doi/10.1103/PhysRevLett.94. 156802>.

[10] A.J. Keller, S. Amasha, I. Weymann, C.P. Moca, I.G. Rau, J.A. Katine, H. Shtrikman, G. Zaránd, D. Goldhaber-Gordon, Emergent SU(4) Kondo physics in a spin-charge-entangled double quantum dot, Nat. Phys. 10 (2) (2014) 145-150 , URL 〈http://dx.doi.org/10.1038/nphys2844〉.

[11] T. Ando, Spin-orbit interaction in carbon nanotubes, J. Phys. Soc. Jpn. 69 (6) (2000) 1757-1763, http://dx.doi.org/10.1143/JPSJ.69.1757, URL 〈http://dx.doi. org/10.1143/JPSJ.69.1757>.

[12] F. Kuemmeth, S. Ilani, D.C. Ralph, P.L. McEuen, Coupling of spin and orbital motion of electrons in carbon nanotubes, Nature 452 (7186) (2008) 448-452, http://dx.doi.org/10.1038/nature06822, URL 〈http://dx.doi.org/10.1038/ nature06822>.

[13] M. Marganska, P. Chudzinski, M. Grifoni, The two classes of low energy spectra in finite carbon nanotubes, Phys. Rev. B, American Physical Society 92(7), 2015, 075433, numpages $=\{9\}, 10.1103 /$ PhysRevB.92.075433, url $=\{$ http://link.aps. org/doi/10.1103/PhysRevB.92.075433\}.

[14] W. Izumida, R. Okuyama, R. Saito, Valley coupling in finite-length metallic single-wall carbon nanotubes, Phys. Rev. B 91 (2015) 235442, http://dx.doi org/10.1103/PhysRevB.91.235442, URL 〈http://link.aps.org/doi/10.1103/Phys RevB.91.235442>.

[15] T.S. Jespersen, K. Grove-Rasmussen, J. Paaske, K. Muraki, T. Fujisawa, J. Nygard, K. Flensberg, Gate-dependent spin-orbit coupling in multielectron carbon nanotubes, Nat. Phys. 7 (4) (2011) 348-353, http://dx.doi.org/10.1038/ nphys1880, URL 〈http://dx.doi.org/10.1038/nphys1880〉.

[16] F.B. Anders, D.E. Logan, M.R. Galpin, G. Finkelstein, Zero-bias conductance in carbon nanotube quantum dots, Phys. Rev. Lett. 100 (2008) 086809, http://dx. doi.org/10.1103/PhysRevLett.100.086809, URL 〈http://link.aps.org/doi/10.1103/ PhysRevLett.100.086809>.

[17] M.R. Galpin, F.W. Jayatilaka, D.E. Logan, F.B. Anders, Interplay between Kondo physics and spin-orbit coupling in carbon nanotube quantum dots, Phys. Rev.
B 81 (2010) 075437, http://dx.doi.org/10.1103/PhysRevB.81.075437, URL 〈http://link.aps.org/doi/10.1103/PhysRevB.81.075437〉.

[18] D.R. Schmid, S. Smirnov, M. Margańska, A. Dirnaichner, P.L. Stiller, M. Grifoni, A.K. Hüttel, C. Strunk, Broken SU(4) symmetry in a Kondo-correlated carbon nanotube, Phys. Rev. B 91 (2015) 155435, http://dx.doi.org/10.1103/PhysRevB.91.155435, URL 〈http://link.aps.org/doi/10.1103/PhysRevB.91.155435〉.

[19] J. Paaske, A. Rosch, P. Wölfle, N. Mason, C.M. Marcus, J. Nygård, Non-equilibrium singlet-triplet Kondo effect in carbon nanotubes, Nat. Phys. 2 (2006) 460-464, http://dx.doi.org/10.1038/nphys340 arxiv:cond-mat/0602581.

[20] C.H.L. Quay, J. Cumings, S.J. Gamble, R.d. Picciotto, H. Kataura, D. GoldhaberGordon, Magnetic field dependence of the spin- $\frac{1}{2}$ and spin-1 Kondo effects in a quantum dot, Phys. Rev. B 76 (2007) 245311, http://dx.doi.org/10.1103/ PhysRevB.76.245311, URL 〈http://link.aps.org/doi/10.1103/PhysRevB.76. 245311>.

[21] A. Makarovski, A. Zhukov, J. Liu, G. Finkelstein, SU(2) and SU(4) Kondo effects in carbon nanotube quantum dots, Phys. Rev. B 75 (2007) 241407, http://dx. doi.org/10.1103/PhysRevB.75.241407, URL 〈http://link.aps.org/doi/10.1103/ PhysRevB.75.241407〉.

[22] A. Makarovski, J. Liu, G. Finkelstein, Evolution of transport regimes in carbon nanotube quantum dots, Phys. Rev. Lett. 99 (2007) 066801, http://dx.doi.org/ 10.1103/PhysRevLett.99.066801, URL 〈http://link.aps.org/doi/10.1103/PhysRev Lett.99.066801>.

[23] J.P. Cleuziou, N.V. N'Guyen, S. Florens, W. Wernsdorfer, Interplay of the Kondo effect and strong spin-orbit coupling in multihole ultraclean carbon nanotubes, Phys. Rev. Lett. 111 (2013) 136803, http://dx.doi.org/10.1103/PhysRevLett.111.136803, URL 〈http://link.aps.org/doi/10.1103/PhysRevLett.111.136803〉.

[24] K. Grove-Rasmussen, S. Grap, J. Paaske, K. Flensberg, S. Andergassen, V. Meden, H.I. Jørgensen, K. Muraki, T. Fujisawa, Magnetic-field dependence of tunnel couplings in carbon nanotube quantum dots, Phys. Rev. Lett. 108 (2012) 176802, http://dx.doi.org/10.1103/PhysRevLett.108.176802, URL 〈http://link. aps.org/doi/10.1103/PhysRevLett.108.176802>.

[25] K.G. Wilson, The renormalization group: critical phenomena and the Kondo problem, Rev. Mod. Phys. 47 (1975) 773-840, http://dx.doi.org/10.1103/RevModPhys.47.773, URL 〈http://link.aps.org/doi/10.1103/RevModPhys.47.773〉.

[26] W. Hofstetter, Generalized numerical renormalization group for dynamical quantities, Phys. Rev. Lett. 85 (2000) 1508-1511, http://dx.doi.org/10.1103/ PhysRevLett.85.1508, URL 〈http://link.aps.org/doi/10.1103/PhysRevLett.85. 1508).

[27] R. Bulla, T.A. Costi, T. Pruschke, Numerical renormalization group method for quantum impurity systems, Rev. Mod. Phys. 80 (2008) 395-450, http://dx.doi org/10.1103/RevModPhys.80.395, URL 〈http://link.aps.org/doi/10.1103/Re vModPhys.80.395〉.

[28] P. Schlottmann, Bethe-Ansatz solution of the Anderson model of a magnetic impurity with orbital degeneracy, Phys. Rev. Lett. 50 (1983) 1697-1700, http //dx.doi.org/10.1103/PhysRevLett.50.1697, URL 〈http://link.aps.org/doi/10.1103 PhysRevLett.50.1697).

[29] K. Yamada, K. Yosida, K. Hanzawa, Comments on the dense Kondo state, Progr Theor. Phys. 71 (3) (1984) 450-457, http://dx.doi.org/10.1143/PTP.71.450, arXiv:http://ptp.oxfordjournals.org/content/71/3/450.full.pdf +html, URL 〈http://ptp.oxfordjournals.org/content/71/3/450.abstract〉.

[30] A.I. Tóth, C.P. Moca, O. Legeza, G. Zaránd, Density matrix numerical renormalization group for non-abelian symmetries, Phys. Rev. B 78 (2008) 245109, http://dx.doi.org/10.1103/PhysRevB.78.245109, URL 〈http://link.aps. org/doi/10.1103/PhysRevB.78.245109).

[31] D.C. Langreth, Friedel sum rule for Anderson's model of localized impurity states, Phys. Rev. 150 (1966) 516-518, http://dx.doi.org/10.1103/PhysRev.150.516, URL 〈http://link.aps.org/doi/10.1103/PhysRev.150.516〉.

[32] M. Eto, SU(4) Kondo effect in quantum dots with two orbitals and spin $1 / 2$, AIP Conf. Proc. 772 (1) (2005) 821-822 http://dx.doi.org/10.1063/1.1994359, URL 〈http://scitation.aip.org/content/aip/proceeding/aipcp/10.1063/1.1994359〉.

[33] A. Carmi, Y. Oreg, M. Berkooz, Realization of the SU $(N)$ Kondo effect in a strong magnetic field, Phys. Rev. Lett. 106 (2011) 106401, http://dx.doi.org/10.1103/ PhysRevLett.106.106401, URL 〈http://link.aps.org/doi/10.1103/PhysRevLett.106. 106401>.

[34] R. Sakano, N. Kawakami, Conductance via the multiorbital Kondo effect in single quantum dots, Phys. Rev. B 73 (2006) 155332, http://dx.doi.org/10.1103/ PhysRevB.73.155332, URL 〈http://link.aps.org/doi/10.1103/PhysRevB.73. $155332\rangle$.

[35] K. Le Hur, P. Simon, D. Loss, Transport through a quantum dot with SU (4) Kondo entanglement, Phys. Rev. B 75 (2007) 035332, http://dx.doi.org/ 10.1103/PhysRevB.75.035332, URL 〈http://link.aps.org/doi/10.1103/PhysRevB. 75.035332〉.

[36] C. Mora, P. Vitushinsky, X. Leyronas, A.A. Clerk, K. Le Hur, Theory of nonequilibrium transport in the SU(N) Kondo regime, Phys. Rev. B 80 (2009) 155322, http://dx.doi.org/10.1103/PhysRevB.80.155322, URL 〈http://link.aps. org/doi/10.1103/PhysRevB.80.155322).

[37] M. Sbaih, M. Srour, Lie algebra and representation of SU(4), Electron. J. Theor Phys. 10 (28) (2013) 9-26 , URL 〈http://ejtp.com/articles/ejtpv10i28p9.pdf〉. 